Reprinted from
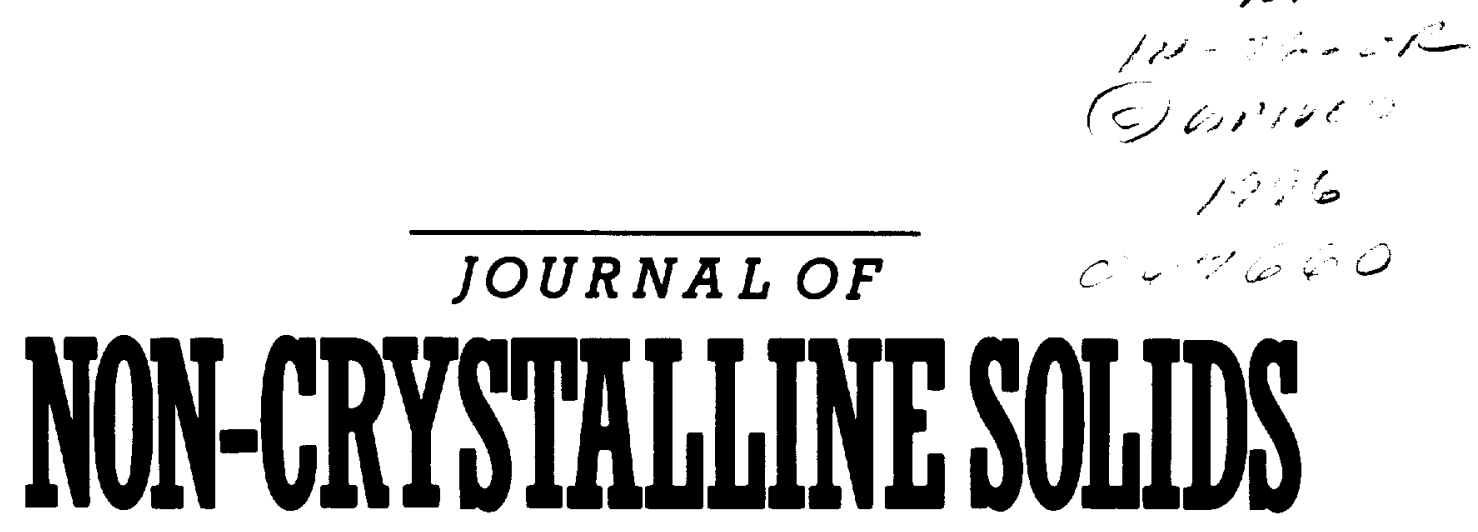

Journal of Non-Crystalline Solids 204 (1996) 1.3-31

\title{
Computer modeling of non-isothermal crystallization
}

\author{
K.F. Kelton ${ }^{a . *}$, K. Lakshmi Narayan ${ }^{a}$, L.E. Levine ${ }^{b}$, T.C. Cull ${ }^{\text {a }}$, C.S. Ray ${ }^{c}$ \\ "Deparment of Physics, Washington Lnicersity, St. Louis, MO 6.3130, USA \\ 'Materials Bldg., B254. NIST. Gathershurg. MD 20899. USA \\ Graduate Conter for Materials Rescarch Uniersity of Missouri-Rolla. Rolla, MO 65401, USA
}

Received 18 September 1995; revised 20) March 1990

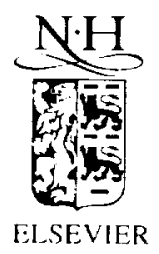




\section{JOURNAL OF NON-CRYSTALLINE SOLIDS}

A journal on the chemical, electronic, optical, and mechanical properties of glasses, amorphous semiconductors and metals, sol-gel materials, the liquid state of these solids, and the processes by which they are formed.

Founding Editor: Professor J.D. Mackenzie

$\begin{array}{lll}\text { Editor: R.A. Weeks } & \text { Co-editor: D.L. Kinser } & \text { Editorial Assistant: A. Cook } \\ +1-6153222923 & +1-6153223537 & +1-6153222058\end{array}$

$+1-615.3222923+$ +1-6153223537

Vanderbilt University, 610 Olin Hall, Nashville, TN 37240, USA

Fax: +1-615343 8645. E-mail: jncs@vuse.vanderbilt.edu

\section{Regional Editors}

G.H. Frischat, Institut für Nichtmetallische Werkstoffe, Technische Universität Clausthal, D-36878 Clausthal-Zellerfeld, Germany G.N. Greaves, Daresbury Laboratory, Daresbury, Warrington WA4 4AD, UK

H. Kawazoe, Tokyo Institute of Technology, Materials and Structures Laboratory, 4259 Nagatsuta, Midori-ku, Yokohama 226, Japan

J. Lucas, Laboratoire des Verres et Céramiques, Université de Rennes I, Ave. du Général Leclerc, Campus de Beaulieu, 35042 Rennes cedex, France

J.H. Simmons. Department of Materials Science and Engineering, University of Florida, 154A Rhines Hall, Gainesville, FL 32611-2066, USA

$\begin{array}{ll}\text { Advisory Editorial Board } & \\ \text { Australia } & \text { People's Rep. of China } \\ \text { D.R. McKenzie, Sydney } & \text { Gan Fuxi, Shanghai } \\ \text { Austria } & \text { Portugal } \\ \text { J. Hafner, Vienna } & \text { R.M. Almeida, Lisbon } \\ \text { Belgium } & \text { Russia } \\ \text { G.J. Adriaenssens, Heverlec-Leuven } & \text { V.I. Arbuzov, St Petersburg } \\ \text { Brazil } & \text { O.V. Mazurin, Si Petersburg } \\ \text { E.D. Zanotto, São Carlos } & \text { Soush Korea } \\ \text { Italy } & \text { U.-C. Paek, Kwangju } \\ \text { A. Montenero, Parma } & \text { Spain } \\ \text { Japun } & \text { M.T. Mora, Barcelona } \\ \text { H. Hosono, Yokohama } & \text { Sweden } \\ \text { N. Soga, Kyoto } & \text { L.M. Torell } \\ \text { K. Tanaka. Ibaraki } & \end{array}$

Taivan

Jenn-Ming Wu, Hsinchu

United Kingdom

S.R. Elliott, Cambridge

A.C. Wright, Reading

USA

S.W. Freiman, Gaithersburg, MD

D.L. Griscom, Washington, DC

K. Kelton, Sı Louis, MO

G.W. Scherer, Wilmington, DE

T.P. Seward III, Corning, NY

M. Tomozawa, Troy, NY

A.K. Varshneya. Alfred, NY

M.C. Weinberg, Tucson, AZ

(for complete addresses, see page 2 of the preliminary pages of issues 1\&2)

\section{Aims and Scope}

Review Articles, Research Papers, Comments and Letters on chemical. electronic, optical, mechanical, structural and fracture properties of non-crystalline materials including glasses, amorphous semiconductors. metals (alloys) and sol-gel materials which form glasses, the liquid state of all materials from which non-crystalline solids can be formed, and the processes for the formation of non-crystalline solids are accepted.

\section{Abstracted/indexed in:}

Biological Abstracts; Ceramic Abstracts; Current Contents: Engineering. Technology and Applied Sciences; El Compendex Plus: Engineered Materials Abstracts; Engineering Index: INSPEC; Metals Abstracts; Physics Briefs.

\section{Subscription Information 1996}

Volumes 192-207 of Joumal of Non-Crystalline Solids
(ISSN 0022-3093) are scheduled for publication. (Frequency: monthly.) Prices are available from the publishers upon request. Subscriptions are accepted on a prepaid basis only and are entered on a calendar-year basis. Issues are sent by SAL (surface air lifted) mail wherever this service is available. Airmail rates are available upon request. Please address all enquiries regarding orders and subscriptions to:

Elsevier Science B.V.

Order Fulfilment Department

PO Box 211, 1000 AE Amsterdam

The Netherlands

Tel: $+31-204853642$, Fax: $+31-204853598$

Claims for issues not received should be made within six months of our publication (mailing) date. If not, they cannot be honoured frec of charge.

US Mailing notice - Joumal of Non-Crystalline Solids (ISSN (0)22-3093) is published monthly, except semimonthly in February, March, May, June, July, August, Septenber and Oclober, by Elsevier Science B.V., Molenwerf 1, P.O. Box 211, 1000 AE Amsterdam, The Netherlands. Annual subscription price in the USA is US\$ 4654 (valid in North, Central and South America only), including air speed delivery. Second class postage paid at Jamaica. NY 11431.

USA Postmasters: Send changes to Journal of Non-Crystalline Solids. Publications Expediting, Inc., 200 Meacham Avenue, Elmont, NY 11003 . Airfreight and mailing in the USA by Publications Expediting.

The paper used in this publication meets the requirements of ANSINISO Z39.48-1992 (Permanence of Paper).

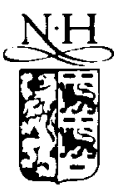




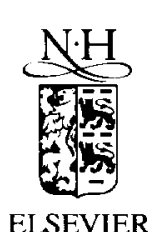

Journal of Non-Crystalline Solids 204 (1996) 13-31

\title{
Computer modeling of non-isothermal crystallization
}

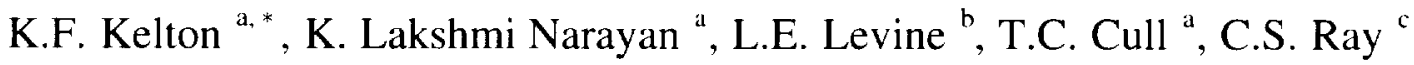 \\ "Department of Physics, Washington Unicersits, St. Louis, MO 6.3130, USA \\ ${ }^{b}$ Materials Bldg. B254. NIST, Gaithersburg. MD 20899. USA \\ "Graduate Center for Materials Research Unitersity of Missouri-Rolla, Rolla. MO 65401. USA
}

Received 18 Septemher 1995; rcvised 20 March 1996

\begin{abstract}
A realistic computer model for simulating isothermal and non-isothermal phase transformations proceeding by homogeneous and heterogeneous nucleation and interface-limited growth is presented. A new treatment for particle size effects on the crystallization kinetics is developed and is incorporated into the numerical model. Time-dependent nucleation rates, size-dependent growth rates and surface crystallization are also included. Model predictions are compared with experimental measurements of DSC/DTA peak parameters for the crystallization of lithium disilicate glass as a function of particle size, Pt doping levels, and water content. The quantitative agreement that is demonstrated indicates that the numerical model can be used to extract key kinetic data from easily obtained calorimetric data. The model can also be used to probe nucleation and growth behavior in regimes that are otherwise inaccessible. Based on a fit to data, an earlier prediction that the time-dependent nucleation rate in a DSC/DTA scan can rise above the steady-state value at a temperature higher than the peak in the steady-state rate is demonstrated.
\end{abstract}

\section{Introduction}

As was demonstrated in a companion paper in this volume [1], * differential scanning calorimetry (DSC) and differential thermal analysis (DTA) provide convenient and rapid methods for studying phase transitions under isothermal and non-isothermal conditions. By carrying out properly designed experiments, the relative importance of surface and volume nucleation can be assessed and some indication of the temperature range of significant nucleation can be obtained. These calorimetric techniques suffer, however, from the paucity of methods for quantita-

\footnotetext{
* Corresponding author. Tel:: + 1-314 935 6228; fax: + 1-314 9356219 ; e-mail: kfk @ wuphys.wustl.edu.
}

tive data analysis. Analysis techniques are typically based on Kissinger-type methods [2,3], which assume an Arrhenius temperature dependence for the transformation kinetics. Since the kinetics are dictated partially by the nucleation rate, which is definitely non-Arrhenius, the use of these methods is questionable when applied to most first-order phase transformations. Experimental [4,5] and theoretical [6] investigations have shown that such techniques can be used with some confidence in transformations involving growth only. There the activation energy obtained is approximately equal to the activation energy for growth. Since this requires that either the primary nucleation be heterogenous and that the sites are saturated, or that the peaks in the nucleation and growth rates be widely separated, these methods have limited applicability. Also, since growth is 
strictly non-Arrhenius, the activation energy obtained will depend on the temperature range over which the transformation took place. This can depend critically on the number of quenched-in nuclei or the rate at which the scan takes place through the nucleation zone, where there will be no accompanying DSC or DTA signal. Clearly, better methods of analysis that can take such effects into consideration are required.

We have developed a realistic numerical computer model for simulating isothermal and non-isothermal phase transformations proceeding by homogeneous and heterogeneous nucleation and interface-limited growth. Unlike earlier models, the nucleation rate need not be steady-state; transient nucleation which is dominant in the formation and crystallization of many silicate and metallic glasses, is included naturally. Further, no ad-hoc assumptions are made of what constitutes a growing cluster contrasted with a nucleation embryo. Cluster evolution is followed directly from the smallest embryo to a macroscopic cluster. Previous comparisons between the model predictions and experimental non-isothermal DSC and DTA data for a polymorphically devitrifying glass, lithium disilicate, are in good agreement. The computer model has been used to demonstrate that all existing methods for analyzing non-isothermal DSC data are flawed [6] and to evaluate a recently proposed method for estimating nucleation rates from DSC and DTA data [7].

Those calculations were made assuming volume nucleation and growth at constant composition in large samples. As was discussed in our companion paper, however, an ability to model finite-size effects and surface nucleation and growth can often be critical for a quantitative analysis of experimental data. Here we extend the numerical model to include these features. It should be emphasized that this is not the first attempt to include finite-size effects and surface crystallization in the analysis of phase transformation kinetics. Surface crystallization has been modeled for both metallic [8] and silicate [9] glasses. These earlier treatments are followed for modeling the surface crystallization here. Finite-size effects on isothermal transformation kinetics have also been considered previously by Weinberg $[10,11]$. The expressions developed there, however, are mathematically complex, making them difficult to extend to non-isothermal transformations. Our simpler expres- sion is more easily used to analyze isothermal transformation kinetic data and is readily adapted to more complicated situations, such as the multi-step isothermal and non-isothermal annealing treatments considered here. The validity of our expression has been established by a quantitative comparison with computer simulations of phase transformations on a lattice of discrete, non-interacting, spins.

To test the extended computer model, we compare the theoretical predictions of DSC/DTA data taken under conditions of non-isothermal heating with the experimental studies of the devitrification of lithium disilicate glass discussed in our companion paper. An important result of this modeling is the confirmation of the importance of time-dependent nucleation in determining DSC/DTA behavior. In particular, the agreement between model predictions and experimental data for isothermal and non-isothermal anneals in the temperature range of significant nucleation supports our previous model prediction that the time-dependent nucleation rate in a DSC/DTA heating scan peaks at a temperature higher than the steady-state rate and rises significantly above the steady-state value there.

\section{Description of the numerical model}

The basic numerical model and the new extensions to include particle size and shape are discussed in this section. Since the basic model has been discussed extensively elsewhere [6,7,12-14], only the most salient points are reviewed here.

\subsection{The basic model for an infinite sample}

The classical theory of nucleation [12] is assumed. taking direct account of time-dependent nucleation rates. Assuming spherical clusters, negligible stress effects and a sharp interface between the cluster and the parent phase, the reversible work of formation for a cluster of $n$ molecules can be written as

$W_{n}=n \Delta G^{\prime}+(36 \pi)^{1 / 3} \bar{l}^{2 / 3} n^{2 / 3} \sigma$.

Here, $\Delta G^{\prime}$ is the Gibbs free energy per molecule of the new phase less that of the initial phase, $\bar{l}$ is the molecular volume, and $\boldsymbol{\sigma}$ is the interfacial energy 
per unit area. Clusters are assumed to evolve slowly in size by a series of bimolecular reactions, leading to a time-dependent cluster distribution described by a system of coupled differential equations of the form

$$
\begin{aligned}
\frac{\mathrm{d} N_{n, t}}{\mathrm{~d} t}= & N_{n-1 . t} k_{n-1}^{+}-\left[N_{n, t} k_{n}^{-}+N_{n, t} k_{n}^{+}\right] \\
& +N_{n+1, t_{n+1},},
\end{aligned}
$$

where $N_{n, 1}$ is the number of clusters of size $n$ at time $t$. The rate of monomer addition and loss, $k_{n}^{+}$and $k_{n}^{-}$respectively, are obtained from the diffusion coefficient in the parent phase and the atomic jump distance (see Refs. [12,13] for details). The nucleation rate at a cluster size $n, I_{n, i}$, is the time-dependent flux of clusters past that size and is given by

$I_{n, t}=N_{n, t} k_{n}^{+}-N_{n+1} k_{n+1}^{-}$.

The coupled differential equations are solved using a finite difference method (see Kelton et al. [13]) in which the time is divided into a large number of small intervals, $\delta t$, and the number of clusters of size $n$ at the end of the interval, $N_{n, t+\delta r}$, is calculated from

$N_{n, t+\delta t}=N_{n, t}+\delta t \frac{\mathrm{d} N_{n, t}}{\mathrm{~d} t}$,

where $\mathrm{d} N_{n, t} / \mathrm{d} t$ is given in Eq. (2).

The behavior of clusters up to some large size is calculated directly by these methods. The growth of clusters larger than this size is computed using an approximate expression for the growth rate as a function of cluster radius, $u(r)$, that was derived previously [14]:

$u(r)=C \frac{16 D}{\lambda^{2}}\left(\frac{3 \bar{v}}{4 \pi}\right)^{1 / 3} \sinh \left[\frac{\bar{v}}{2 k_{\mathrm{B}} T}\left(\Delta G_{v^{\prime}}-\frac{2 \sigma}{r}\right)\right]$.

Here $D$ is the diffusion coefficient in the parent phase, $\lambda$ is the jump distance, $\Delta G_{v}$ is the Gibbs free energy per unit volume, $T$ is the temperature, $k_{\mathrm{B}}$ is Boltzmann's constant, and $C$ is a constant that is dependent on the mode of growth [15].

To simulate glass formation and DSC non-isothermal scans, the rate of volume fraction transformed under non-isothermal conditions is computed by dividing the time into a series of isothermal scans of duration $\delta t=\delta T / \Phi$, where $\Phi$ is the scan rate in degrees per second and $\delta T$ is the temperature step size, allowing the nucleation rate to evolve in each interval as described by Eqs. (2) and (4). At the end of the interval, the sizes of the nuclei generated in previous intervals are calculated using the average growth rate, $u(r)$, given by $\mathrm{Eq}$. (5). The extended volume fraction transformed, $x_{c}$, is calculated at the end of each interval:

$x_{\mathrm{c}}=\frac{1}{V_{\mathrm{o}}} \sum_{i=1}^{m}\left(\frac{4 \pi}{3}\right) N_{i} r_{i, 1}^{3}$,

where $N_{i}$ is the number of nuclei generated in the interval $I, r_{i, l}$ is the time-dependent radius of those nuclei, and $V_{0}$ is the sample volume. Assuming that the nucleation and growth occur randomly in space, the actual volume fraction, $x(t)$, is computed assuming Johnson-Mehl-Avrami-Kolmogorov (JMAK) statistics [16-18]:

$x(t)=1-\exp \left(-x_{\mathrm{e}}\right)$.

Assuming a linear relation between the rate of change of the enthalpy, $\mathrm{d} H / \mathrm{d} t$, and the rate of volume fraction transformed, the DSC trace is computed as a function of temperature or time as

DSC signal $\alpha \frac{x\left(T_{i}+\delta T\right)-x\left(T_{i}\right)}{\delta t}$.

\subsection{Model extensions}

The model discussed in the previous section assumes (1) that the sample is large compared with the size of an individual grain of the daughter phase, (2) that nucleation occurs randomly in space and time and (3) that the growth is three dimensional with the grains of the daughter phase having a low aspect ratio. Devitrification in real samples, however, often occurs under quite different conditions. Surface crystallization, for example, is common in silicate glasses, although internal, or volume, nucleation and growth usually dominates in bulk glasses since the surface constitutes a small fraction of the sample. Samples used for calorimetric studies, however, are generally fine-grained powders (typically 50 to $400 \mu \mathrm{m}$ in diameter) with a very large surface to volume ratio. Surface growth, if it occurs, will then contribute more substantially to the transformation signature. 
Also, since a cluster nucleates within a particle, all space is not available for growth; the region outside the particle is excluded, thus violating a fundamental assumption of the JMAK statistics. In this section, we present approximate methods for dealing with these effects in isothermal and non-isothermal transformations. Their successes and limitations are discussed in Section 3.

\subsubsection{Finite size (FS) effects}

The probable number of nuclei appearing in a small particle of volume $V_{\mathrm{p}}$, given the number expected in a mole of volume $V_{\text {nole, }}$ is $N_{\mathrm{p}} \approx$ $N_{\text {nule }}\left(V_{\mathrm{p}} / V_{\text {mole }}\right)$. Expressed in terms of the nucleation rate, $I_{\text {mole }}$, then the average number of nuclei per particle appearing in a given time interval, $\Delta t$, is

$N_{\mathrm{p}}=I_{\text {mole }} \frac{V_{\mathrm{p}}}{V_{\text {mole }}} \Delta t$.

The distribution of nuclei among the particles of the ensemble, given $N_{\mathrm{p}}$ average nuclei per particle, is calculated from the Poisson distribution:

$P\left(n_{\mathrm{p}}\right)=\mathrm{e}^{-N_{\mathrm{r}}} \frac{N_{\mathrm{p}}^{n_{\mathrm{r}}}}{n_{\mathrm{p}} !}$,

where $n_{p}$ is the number of nuclei per particle. To minimize the calculation time in the computer modeling, slightly different approaches were taken depending on the size of $N_{\mathrm{p}}$. For $N_{\mathrm{p}}<300$, Eq. (10) was applied for each value of $n_{p}$ between 0 and $5 N_{p}$. a value sufficiently large that $P\left(5 N_{\mathrm{p}}\right)<1$. For 300 $<N_{\mathrm{f}}<10000$, the particles were partitioned into bins of width $\Delta$ (set by the constraint that the total number of bins was 1500) and Eq. (10) was used to compute $P(n)$, taken as the average value over the bin (i.e., $\langle P(n)\rangle)$. For $N_{p}>10000$ the distribution was sufficiently narrow that statistical fluctuations among particles could be ignored.

Growth presents different problems. Given a single nucleation event within a particle of radius $R$, a cluster will grow with a cluster-dependent growth velocity given by $\mathrm{Eq}$. (5). If the cluster were to nucleate at the exact center of the particle and if there were no other growing clusters within the particle. the transformation would proceed unimpeded until the particle was completely transformed. For clusters nucleated away from the center, how-
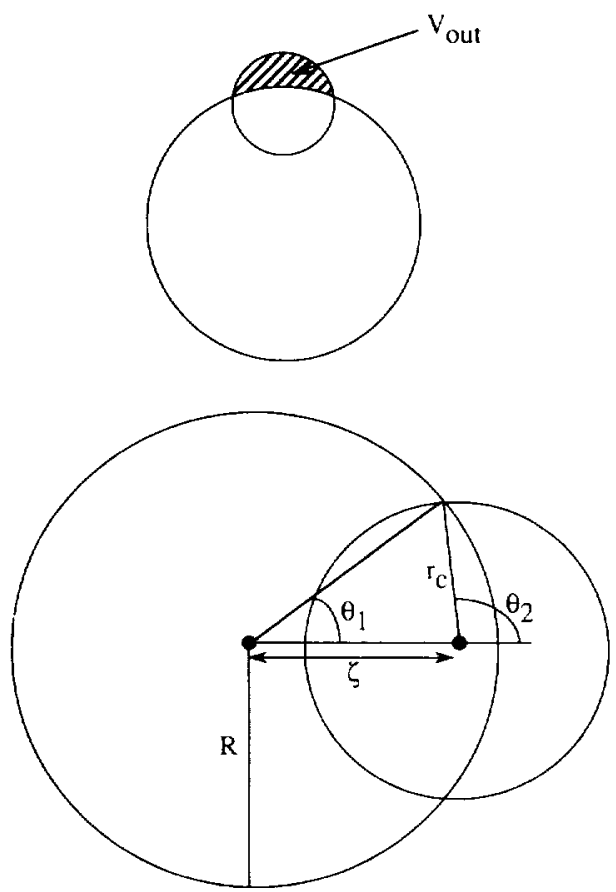

Fig. 1. Schematic figure illustrating the case of a spherical nucleus growing in a finite-sized particle.

ever, the transforming region will intersect the particle surface prior to the complete transformation of the particle. Now the assumption of unimpeded growth requires that some of the transformed volume fall outside the boundary of the particle (labeled as $V_{\text {rut }}$ in Fig. 1). Clearly this region cannot contribute to the actual volume fraction transformed. To model such finite size effects, we assume that (1) the clusters form randomly in space and time and grow independently of each other, (2) the number of nuclei produced in a particle is proportional to the volume of the particle (Eqs. (9) and (10)) and (3) growth outside the particle is prohibited. Since finite computer resources make it impossible to calculate the positions of all clusters in all particles and to follow their growth directly, a statistical description is developed which gives the average growth volume outside a particle in terms of the cluster and particle radii, $r_{\mathrm{C}}$ and $R$ respectively. This volume, $V_{\text {oul }}\left(r_{\mathrm{C}}\right.$ $R$ ), is then subtracted from the extended volume transformed before applying the JMAK statistics (Eq. (7)). 
For simplicity, we assume spherical particles of radius $R$. Given a cluster of radius $r_{\text {c }}$ with a center at some position $\zeta \leq R$, the volume of the cluster falling outside the boundary of the particle is

$$
\begin{aligned}
V^{\prime}\left(R, r_{\mathrm{C}}\right)= & \frac{\pi}{3} r_{\mathrm{C}}^{3}\left(1-\cos \theta_{2}\right)^{2}\left(2+\cos \theta_{2}\right) \\
& -\frac{\pi}{3} R^{3}\left(1-\cos \theta_{1}\right)^{2}\left(2+\cos \theta_{1}\right),
\end{aligned}
$$

where $\theta_{1}$ and $\theta_{2}$ are defined as shown in Fig. 1. Three cases are relevant to the calculations presented, corresponding to different positions and relative sizes of the growing cluster and particle: (i) $\theta_{1}$ and $\theta_{2}<90^{\circ}$; (ii) $\theta_{1}<90^{\circ}$ and $\theta_{2}>90^{\circ}$ : and (iii) $\theta_{1}$ and $\theta_{2}>90^{\circ}$. An application of the law of cosines demonstrates that for all three cases $V^{\prime}\left(R, r_{c}\right)$ is given by Eq. (11). These results can be extended directly to the case of clusters growing in a large number of particles of equal size. constituting a powder sample, such as is often used in DSC or DTA experiments. For a cluster with a radius smaller than the particle in which it is growing to have predicted growth outside the particle. the cluster's center must be located within a shell of inner radius $R-r_{C}$ and outer raldius $R$. Assuming the internal nucleation to be equally probable in all regions, the probability that the center of a given cluster lies between a radius $\zeta$ and $\zeta+\mathrm{d} \zeta$ in the particle is

$P(\zeta, \zeta+\mathrm{d} \zeta)=\frac{4 \pi \zeta^{2} \mathrm{~d} \zeta}{\left(4 \pi R^{3}\right) / 3}=\frac{3 \zeta^{2}}{R^{3}} \mathrm{~d} \zeta$.

The average value for the volume lying outside the particle, $V_{\text {out }}\left(R, r_{C}\right)$ can he obtained by integrating $V^{\prime}\left(R, r_{C}\right)$ over all possible locations of the center of the growing cluster in the particle. weighing by the factor in Eq. (12). Since clusters with centers farther than $r_{C}$ from the particle surface grow entirely within the particle, they will not contribute to the volume outside the particle; hence the cluster center locations of interest lie within the bounds $R-r_{\mathrm{C}}$ to $R$. giving

$$
\begin{aligned}
& V_{\text {out }}\left(R, r_{\mathrm{C}}\right) \\
& \quad=\frac{\pi r_{\mathrm{C}}^{3}}{R^{3}} \int_{R-r_{\mathrm{c}}}^{R}\left[1-\left(\frac{R^{2}-r_{\mathrm{C}}^{2}-\zeta^{2}}{2 r_{\mathrm{C}} \zeta}\right)\right]^{2}
\end{aligned}
$$

$$
\begin{aligned}
& \times\left[2+\left(\frac{R^{2}-r_{C}^{2}-\zeta^{2}}{2 r_{c} \zeta}\right)\right] \zeta^{2} \mathrm{~d} \zeta \\
& -\pi \int_{R-r^{2}}^{R}\left[1-\left(\frac{R^{2}-r^{2}+\zeta^{2}}{2 R \zeta}\right)\right]^{2} \\
& \times\left[2+\left(\frac{R^{2}-r^{2}+\zeta^{2}}{2 R \zeta}\right)\right] \zeta^{2} \mathrm{~d} \zeta
\end{aligned}
$$

Because the weighing increases strongly with increasing $r$, the lower limit can be extended to $r=0$ without significant error, leading to

$V_{\text {rut }}\left(R, r_{\mathrm{C}}\right)=\frac{\pi r_{\mathrm{C}}^{4}}{24}\left[\frac{18 R^{2}-r_{\mathrm{C}}^{2}}{R^{3}}\right]$.

This formulation is correct for all cases where $0 \leq r_{C}$. $\leq 2 R$. Clearly, for those cases where the growing cluster has a radius larger than that of the particle ( $R \leq r_{\mathrm{C}} \leq 2 R$ ), the lower limit of the integral must be set to zero to avoid double counting. Values of $V_{\text {out }}\left(R, r_{\mathrm{C}}\right)$ computed from Eq. (14) agreed quantitatively with the results from a direct computer calculation of the average cluster volume lying outside the particle, for a set of clusters of given size that were located randomly in an ensemble of 10000 spheres. For more than one growing cluster, the average value for $V_{\text {out }}\left(R, r_{\mathrm{C}}\right)$ per particle must be obtained by summing the separate contributions of all growing clusters:

$V_{\text {out }}^{\mathrm{T}}=\sum_{\text {ill clusters }} V_{\text {tout }}\left(R, r_{\mathrm{C}}\right)$.

where $V_{\text {uut }}\left(R, r_{\mathrm{C}}\right)$ is the average volume lying outside the particle for a cluster of radius $r_{C}$. For the purposes of the calculation, then, the volume transformed, taking into account that which lies outside the particle but not accounting for overlap of clusters within the particle, is

$V=\sum_{\text {all clusters }}\left(\frac{4 \pi}{3} r_{\mathrm{C}}^{3}-V_{\text {out }}\left(R, r_{\mathrm{C}}\right)\right)$.

and the 'corrected' extended volume fraction transformed, $x^{\prime}$, is

$x^{\prime}=\frac{V}{V_{\text {pirt }}}=\frac{V}{\left(4 \pi R^{3}\right) / 3}$. 
A powder will actually contain a distribution of particle sizes, which can be incorporated easily into the calculation. A series of equations having the same form as Eqs. (16) and (17) is used, one for each particle size. $R_{i}$, giving

$V=\sum_{\substack{\text { all clusters } \\ \text { all particless }}}\left(\frac{4 \pi}{3} r_{\mathrm{C}}^{3}-V_{\text {out }}\left(R . r_{\mathrm{C}}\right)\right)$.

The corrected extended volume fraction transformed is changed in a similar manner:

$x^{\prime}=\frac{V}{\sum_{\text {ill partickis }}\left(4 \pi R^{3}\right) / 3}$.

The computer simulation of the transformation now proceeds as for the infinite-sized particles, using Eqs. (16) or (18) to compute the extended volume fraction transformed in each iterative step and computing the corrected extended volume fraction transformed using Eqs. (17) or (19). Overlaps between different clusters are assumed to obey the standard JMAK statistics (Eq. (7)). giving the volume fraction transformed as

$x=1-\exp \left(-x_{0}^{\prime}\right)$.

The DSC/DTA signal is then computed using Eq. (8).

It is important to emphasize that this method for incorporating finite particle sizes still uses the JMAK method to account for cluster overlap. That analysis hinges on the assumption that the nucleation and growth occurs randomly within the sample and that the size of each transformed cluster is small compared with the sample volume. Because of this last point, the analysis developed in this section should be valid when the number of nuclei in each cluster is large. A high nucleation rate during the scan or a large number of quenched-in nuclei is therefore required. Unfortunately, this condition is frequently not true in silicate glasses, given the relatively low nucleation rates. A further extension of this analysis is discussed in the next section.

\subsubsection{Corrections to finite-size calculation for small numbers of nuclei}

For small particles, when the number of nuclei is less than 5 to 10 per particle, non-isothermal calcula- tions made following the approach discussed in Section 2.2.1 predict two DSC peaks, which is in conflict with the experimental data, showing only one peak for all particle sizes. This is an artifact arising from a failure of Eqs. (13)-(20) to properly account for the excluded volume when the volume of each transforming region becomes a significant fraction of the particle volume. This was confirmed recently from a comparison between the predictions of the time-dependent volume fraction transformed by isothermal annealing and computer simulations of the transformation of a finite ensemble of non-interacting spins on a lattice [19]. Those results for the volume fraction transformed are reproduced in Fig. 2 for a scaled time, $\kappa$,

$\kappa=u t / R$,

where $u$ is the growth velocity of the cluster and $R$ is the particle radius. For two nuclei per particle

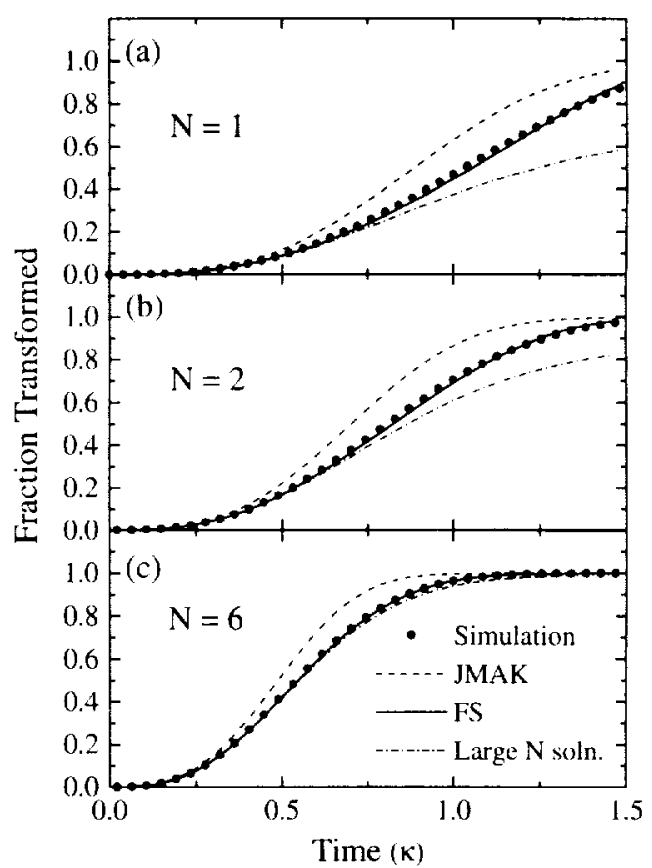

Fig. 2. The fraction transformed as a function of reduced time, $\kappa$, for (a) one, (b) two and (c) six crystallites in a particle. The simulation results were obtained on a spherical lattice of radius 50 units. The uncorrected JMAK (Eqs. (6) and (7)), finite-size corrected (FS) (Eq. (25) and large $N$ (Eqs. (19) and (20)) solutions are shown. The finite-size corrected solution provides the best fit to the lattice simulation. Taken from Ref. [19]. 
$(N=2)$, the volume fraction transformed predicted from Eqs. (16), (17) and (20) fits the lattice simulation well to $x \approx 0.5$, but diverges markedly above that value, approaching a value less than complete transformation. As we will show, this incorrect asymptotic behavior is the origin of the predicted double peak in the DSC scan. The agreement between the simulation and predicted results improves quickly with increasing number of nuclei per particle, with the asymptotic value better approaching the correct value of 1 . The simulation results and the predicted behavior from Eqs. (16), (17) and (20) are in almost perfect agreement for $N>6$. Because of the low nucleation rates in silicate glasses, it is easily possible to produce powders of sufficiently small size that the number of growing clusters per particle is less than 6. To accurately model the transformation behavior, then, it is desirable to extend the analytical expression for finite-size effects to those cases.

For $N$ spherical crystallites of the same size growing in a spherical particle, the approximate expression developed in Section 2.2.1 for the transformed volume fraction, taking account of overlap, can be rewritten as (i.e., combining Eqs. (14), (16), (17) and (20))

$x=1-\exp \left[-N\left(\kappa^{3}-\frac{9}{16} \kappa^{4}+\frac{1}{32} \kappa^{\kappa}\right)\right]$.

As shown, this provides an excellent description for $x(t)$ when the number of clusters per particle, $N$, is greater than 5 to 10 . To extend it to smaller sizes, it is useful to consider the extreme case of $N=1$, for which Eq. (22) provides a poor description (Fig. 2). For the case of one crystallite growing in a spherical particle, there is no overlap, and Eqs. (14) and (16) may be used directly (recasting here for the scaled time),

$x=\kappa^{3}-\frac{9}{16} \kappa^{4}+\frac{1}{32} \kappa^{6}$,

which can be expanded to the same form as Eq. (22):

$$
=1-\exp \left[-\left(\kappa^{3}-\frac{9}{16} \kappa^{4}+\frac{17}{32} \kappa^{6}-\frac{9}{16} \kappa^{7}+\ldots\right)\right] \text {. }
$$

Surprisingly, even for the extreme case of $N=1$, Eqs. (22) and (24) are identical up to the $\kappa^{6}$ term. Based on this, Levine et al. [19] demonstrated that Eq. (24) can be modified simply to describe finite- size effects for all particle sizes, making the coefficient of the $\kappa^{6}$ term a function of the number of crystallites per particle and dropping higher-order terms:

$$
x=1-\exp \left[-N\left(\kappa^{3}-\frac{9}{16} \kappa^{4}+\frac{m(N)}{32} \kappa^{6}\right)\right] .
$$

Fitting the predictions of Eq. (25) to the results of the lattice simulation gave

$m(N)=\max (5.26-0.26 N, 1.0)$.

The quality of the fits from Eq. (25) are shown in Fig. 2; the computed values for $x(t)$ are virtually indistinguishable from the lattice simulation results. Two additional points should be made: (1) the $x(t)$ computed from the JMAK solution becomes better as $N$ increases, although it does so slowly (only approaching within $1 \%$ of the correct value at halftransformation when $N \approx 42,000$ [19]) and (2) Eq. (25) is valid even for a single nucleus growing in a particle, where overlapping volume need not be considered. Eq. (25) then provides a simple expression for taking account of finite size transformation effects over an astonishingly large range. As was demonstrated previously [19], Eq. (25) can also be modified easily to take particle shape into account by substituting for $\kappa$ an effective value, $\kappa^{\mathrm{e}}$ :

$\kappa^{\mathrm{c}}=\left(\frac{V_{\mathrm{c}}}{V}\right)^{1 / 3}$,

where $V_{\mathrm{c}}$ is the extended volume of a growing crystallite and $V$ is the volume of the particle. Eq. (27) was used to convert the transformation data computed for an infinite sample size (cf. Section 3.1.). Since for lithium disilicate glass the nucleation and growth regions are well separated in temperature, all crystallites will be approximately the same size. The reduced time, $\kappa^{c}$, is then directly obtained from the extended volume fraction transformed for an infinite system $x_{x}^{\mathfrak{C}}(t)$ :

$\kappa^{c}=\left(\frac{x_{x}^{2}(t)}{N}\right)^{1 / 3}$

An analysis of the asymptotic value of Eq. (22) reveals the origin of the double peaks predicted in the DSC calculations when following the method 
outlined in Section 2.2.1. That development is only valid for $\kappa \leq 2$, corresponding to the maximum time required for a single cluster to transform the particle. From Eq. (22) that time corresponds to $x=1-\mathrm{e}^{-N}$, the asymptotic value observed in Fig. 2, showing why the agreement between the predicted $x(t)$ and the lattice simulation results improves rapidly with increasing $N$. As $\kappa$ increases without bound, Eq. (22) will eventually approach 1 , although this is outside the region of its validity. The first predicted peak, then, corresponds to the marked decrease in the rate of transformation corresponding to the first asymptote; the second peak arises because of the exponentially increasing growth velocity with temperature. carrying the expression to the second, although non-physical, asymptote. As expected, since Eqs. (25) and (26) give the correct asymptotic behavior (Fig. 2), only single DSC/DTA peaks are predicted.

\subsubsection{Surface crvstallization}

As demonstrated in our companion paper, surface crystallization is appreciable in lithium disilicate glass. Owing to the increase of the surface-to-volume ratio, it can dominate the crystallization behavior of the small particles generally used in DSC/DTA experiments. The magnitude and temperature dependence of the surface nucleation rates are required to model this process, although for a polymorphic transformation, the crystal growth rate should be the same as that used for internal growth. Optical microscopy showed that in the undoped or nonnucleated glasses, a thin, complete, surface layer grew toward the particle center, indicating that the surface nucleation rate is extremely high in this temperature range. For the purposes of modeling, therefore, we ignore the nucleation step and calculate only the rate of transformation due to a spherical growth front originating from the surface.
Assuming a constant growth velocity, $u$, the volume fraction transformed by surface growth alone is

$x_{s}=\left[1-\left(\frac{R-u t}{R}\right)^{3}\right]$.

Experimentally, surface and volume crystallization proceed simultaneously, making it necessary to account for overlap between the two processes as

$x(t)=x_{s}+x_{v}\left(1-x_{s}\right)$.

Here $x_{\mathrm{v}}$ is the fraction transformed from volume nucleation and growth. This approach is similar to previous treatments $[8,9]$.

\subsection{Input parameters}

Several parameters were required to model the experimental data for the crystallization of lithium disilicate glass. The free energy was calculated as a function of temperature, $T$ [20]:

$\Delta G=a_{0}+a_{1} T+a_{2} T^{2}+a_{3} T^{3}$.

A temperature dependent interfacial energy was obtained by fitting the measured steady-state nucleation rates [21]

$\sigma=\sigma_{0}+\sigma_{1} T$.

The viscosity of the glass was assumed to follow a Fulcher-Vogel form:

$\eta=\eta_{0} \exp \left(\frac{\xi}{T-T_{0}}\right)$

where $\eta_{0}, \xi$, and $T_{\mathrm{v}}$ are estimated from experimental data [22]. The value for $\eta_{w}$, was refined by fitting the measured transient times for homogeneous nucleation [21], keeping the temperature dependence fixed at the value obtained from the viscosity data. The

Table 1

\begin{tabular}{llllll}
\hline Gibbs free energy $(\mathrm{J} / \mathrm{mol})$ & Ref. [20] & $a_{0}=48045$ & $a_{1}=-36.81$ & $a_{2}=0.005607$ & $a_{3}=-4.3179 \times 10^{-6}$ \\
Interficial energy $\left(\mathrm{J} / \mathrm{m}^{2}\right)$ & Ref. [21] & $\sigma_{0}=0.094$ & $\sigma_{1}=7 \times 10^{-5}$ & \\
Viscosity $(\mathrm{P})$ & Ref. [22] & $\eta_{0}=0.0363$ & $\xi=3.370$ & $T_{0}=460$ \\
Atomic volume $\left(10^{-6} \mathrm{~m}^{3} / \mathrm{mol}\right)$ & Ref. [23] & $r=61.2$ & & \\
Jump distance $(\mathrm{A})$ & Ref. [21] & $\lambda=4.6$ & & \\
\hline
\end{tabular}


diffusivity was calculated from the Stokes-Einstein relation

$D=\frac{k_{\mathrm{B}} T}{3 \pi a \eta}$,

where $a$ is a characteristic distance of order of the atomic diameter. The values of the parameters used are listed in Table 1.

The value of $C$ in Eq. (5) was determined by requiring agreement between the calculated and measured $[15,24]$ values for the macroscopic growth rate in lithium disilicate glass. The value, $C \approx 4.8$, so determined was held fixed for all calculations presented here.

\section{Results and discussions}

The numerical approach was tested by comparing model predictions for DTA/DSC studies with experimental data from lithium disilicate $\left(\mathrm{LS}_{2}\right)$ glasses. Glasses prepared with different quench rates have different numbers of quenched-in nuclei and different cluster-size distributions. The simulated glasses used in these studies were quenched on the computer following a procedure described elsewhere [14]; unless stated otherwise, a quench rate of $1^{\circ} \mathrm{C} / \mathrm{s}$ was assumed. Possible quench-rate effects on the atomic mobility are not considered. The simulated asquenched glasses were annealed on the computer, under both isothermal and non-isothermal conditions, and scanned to higher temperatures at different rates to simulate a DSC/DTA scan. The results are presented in this section.

\subsection{Particle size effects}

As discussed in our companion paper [1], the DSC /DTA peak parameters are a strong function of the particle size. To investigate the validity of the numerical model, DSC/DTA peaks for the asquenched glasses were computed at a scan rate of $15^{\circ} \mathrm{C} / \mathrm{min}$ for several particle radii: $17.5 \pm 5 \mu \mathrm{m}$, $45 \pm 8 \mu \mathrm{m}, 120 \pm 30 \mu \mathrm{m}, 231 \pm 19 \mu \mathrm{m}$, and $510 \pm$ $85 \mu \mathrm{m}$. These size ranges are the same as those used in the experimental measurements [1]. A uniform size distribution was assumed.

\subsubsection{Spherical particles}

Given an ensemble of small particles, if the nucleation rate is low it is likely that some particles will never develop internal nuclei. The statistical nature of this nucleation step was treated following the procedure discussed in Section 2.2.

The results from calculations for spherical particles undergoing finite-size volume (FS) and surface crystallization are compared with experimental data in Fig. 3, showing the peak temperature, $T_{\mathrm{p}}$ (a) and the peak height (b) as a function of particle size. For comparison, the predicted behavior for the surface (Eq. (29)) and volume (FS, Eq. (25)) contributions and the asymptotic values for the infinite sample are also shown. There is little difference between the surface and surface + volume calculations of the peak temperature; both agree well with the experimental data. Although the magnitude of change in the peak temperature predicted from internal nucleation alone

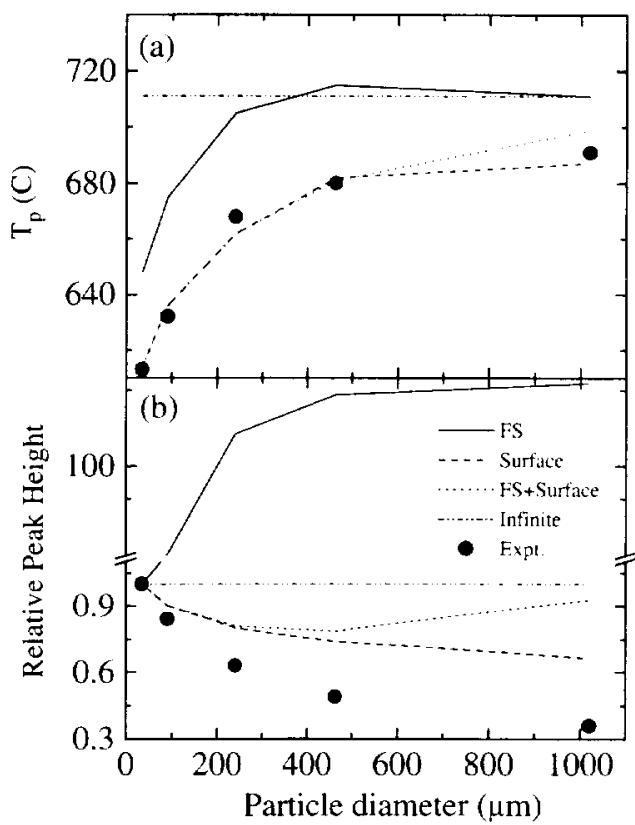

Fig. 3. Effect of particle size on (a) the DSC /DTA peak temperature and (b) the relative peak height (height normalized to that of the $35 \mu \mathrm{m}$ particles). (Note that the upper part of the split vertical axis for (b) is logarithmic while the lower part is linear.) Calculated results for an infinite sample, finite-size corrected (FS), surface crystallization and a combination of FS and surface corrections are shown. All calculations were made for spherical glass particles that were quenched at $1{ }^{\circ} \mathrm{C} / \mathrm{s}$. 
(FS) disagrees with the data, the trend with particle size is correct. Both the magnitude and direction of the change are incorrectly predicted for the peak height. however. This strong effect is a result of the low volume nucleation rate in these glasses; it rethects the statistical absence of nuclei in a sizable fraction of the small particles. Without surface crystallization, those particles do not crystallize and hence do not contribute to the DSC/DTA signal. Larger particles have a higher probability of developing at least one nucleus. Consequently, a larger volume fraction will transform, resulting in a larger signal. This also explains why the peak temperature rises slightly above the infinite particle value for intermediate particle sizes. The excellent agreement between the experimental data for small particles and the calculation based only on surface crystallization, and the near indistinguishability of the surface and surface + volume calculations for these small particles reflects the decreased importance of internal growth due to the large surface-to-volume ratio. Only at the largest particle sizes do the surface and surface + volume calculations begin to differ; both however follow the general trend of the data.

These calculations show a broad minimum in the DSC/DTA peak height near particle diameters of $400-600 \mu \mathrm{m}$. which becomes more prominent when the calculations are carried out to larger particle sizes (Fig. 4(a)). Three regions can be defined as a function of particle size; they reflect behavior arising from a decreasing surface-to-volume ratio with increasing particle size. Region 1, occurring before the minimum in peak height, is dominated by surface crystallization. Both surface and internal crystallization are important in region 2 immediately following the minimum and extending to the point where the curve has nearly reached its asymptotic (infinite volume) value. Volume nucleation and growth is dominant for the large particle sizes of region 3 . A similar, although less dramatic, crossover behavior is also predicted for the peak temperature (Fig. 4(b)). The continuing increase in peak temperature with particle size for the surface calculation simply reflects the decreasing surface-to-volume ratio. The strong effect predicted for the DSC /DTA peak height makes it a potentially useful parameter for ascertaining at what size volume nucleation begins to dominate, allowing a rough assessment of the importance

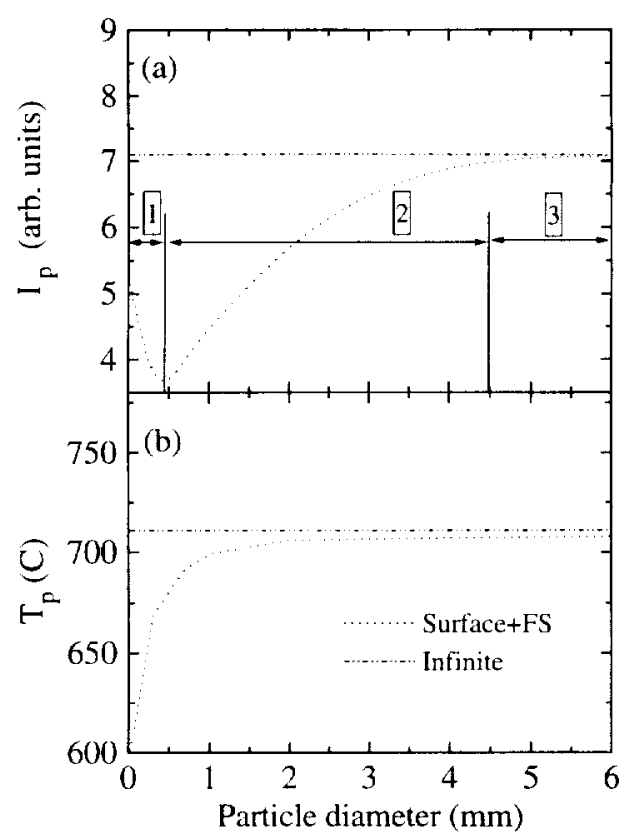

Fig. 4. Calculated results for finite-size corrected (FS) plus surface crystallization and an infinite sample (a) for the DSC/DTA relative peak height, $I_{p}$, (height normalized to that of the $35 \mu \mathrm{m}$ particles) and (b) the peak temperature. Three distinct regions are evident in the behavior of the peak height: surface crystallization dominates in zone 1, surface and volume crystallization contribute in zone 2 , volume crystallization dominates in zone 3. The glass was quenched at $1{ }^{\circ} \mathrm{C} / \mathrm{s}$.

of surface crystallization. Although the predicted minimum in the peak height is not observed experimentally, the data were not taken for sufficiently large particle sizes that the effect becomes pronounced.

\subsubsection{Ellipsoidal particles}

For simplicity, spherical particles were considered for most calculations. Optical and electron microscopy studies, however, show that the particles have a range of shapes as well. To investigate possible effects due to this, changes in the DSC/DTA peak profiles were computed for ellipsoids. Fig. 5 compares the predictions for the ellipsoidal and spherical particles as a function of particle size. While the functional dependence of the peak temperature on particle size is virtually unchanged, the maximum in the peak width (not shown) and the minimum in the peak height (Fig. 5(b)) are shifted to 


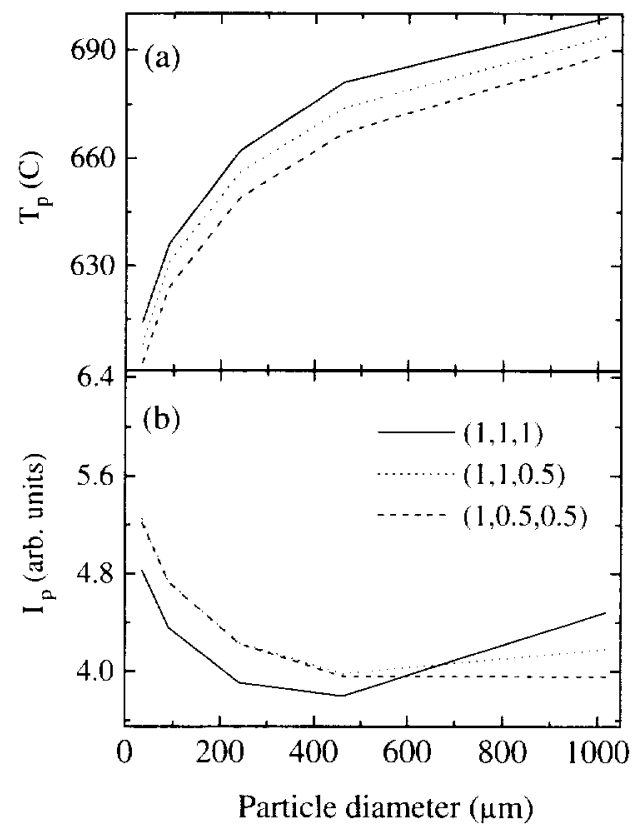

Fig. 5. The calculated effect of particle shapes on (a) the DSC/DTA peak temperature and (b) the relative peak height. $l_{\mathrm{p}}$. The legend indicates the aspect ratios of the ellipsoidal particles considered. The glass was quenched at $1{ }^{\circ} \mathrm{C} / \mathrm{s}$.

larger cluster sizes and are more shallow for the more eccentric particles.

\subsubsection{Heterogeneous nucleation}

Support for the validity of these computer predictions is found in the changes reported in our companion paper [1] for the DSC/DTA peak parameters with the density of nucleating agents in heterogeneously nucleating glasses. The peak height decreased monotonically with increasing particle size for pure lithium disilicate glass; it initially decreased but subsequently increased dramatically with increasing particle size in glasses that had been heavily doped with $\mathrm{Pt}$. Using thermodynamic and kinetic parameters determined from nucleation measurements of the Pt-induced heterogeneous nucleation rates [25], values for $T_{\mathrm{p}}$ and peak height were computed as a function of spherical particle diameter for pure lithium disilicate glass and for glasses containing $1 \mathrm{ppm}$ and $5 \mathrm{ppm}$ Pt impurities (Fig. 6(a) and (b)). The peak profile is dominated by surface crystallization for small particles and is independent of the amount of Pt added to the glass. For larger particles, however, a lower asymptotic temperature is obtained with increasing $\mathrm{Pt}$ dopant level, reflecting the accelerated transformation due to the larger number of internal nuclei. Similarly, the peak height goes through a minimum that occurs earlier and is sharper in the more heavily doped glasses. Although the trends are predicted correctly, the calculated results disagree in detail with the experimental data; in particular the sharp increase experimentally observed in the heavily doped ( $5 \mathrm{ppm}$ ) glass is not reproduced and the predicted rise in peak height for the $1 \mathrm{ppm}$ doped glass is not observed.

The predicted doping dependence of the peak temperatures for ellipsoidal particles has the same form with increased particle size as that shown in Fig. 6(a), although the magnitudes are different. As expected, the effect on the peak height is more dramatic (Fig. 6(c)). Significantly better agreement is

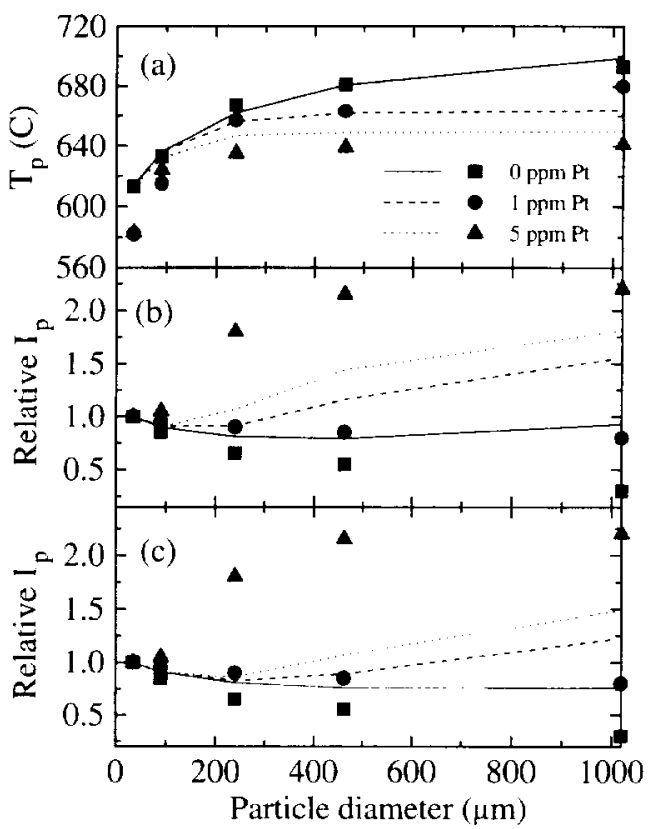

Fig. 6. The predicted effect of Pt doping on (a) the peak temperature and (b) the relative peak height, $I_{p}$, for spherical particles. Figure (c) shows the predicted change in the relative peak height for ellipsoidal particles of aspect ratio $\left(1, \frac{1}{2}, \frac{1}{2}\right)$. The legend indicates the amount of $\mathrm{Pt}$ introduced by weight: the points indicate the experimental dala: the lines represent the computed result. All calculations were performed for glasses quenched at $1{ }^{\circ} \mathrm{C} / \mathrm{s}$ 
obtained between the computer predictions for the ellipsoidal particles and the experimental data on the undoped and $1 \mathrm{ppm}$ doped glasses. The heavily doped glasses, however, still show a sharper increase than is predicted from the calculations. This suggests that the assumption of ellipsoidal particles alone still may not adequately capture the range of particle shapes. More detailed comparisons would require tighter control of the particle shape distribution of the glasses.

\subsubsection{Effects of water}

Water is known to have a profound effect on the stability of silicate glasses [26], presumably due to an increase in the atomic mobility. This was evidenced by a difference in the experimentally observed DSC/DTA peak profile parameters for 'dry' glasses versus 'wet' glasses [1]. A lower transition temperature and a higher peak height were reported in glasses that were kept in a humid environment for extended times. Since the diffusion of water into the sample occurs from the surface, the enhanced surface crystallization is expected to have the greatest impact on the transformation kinetics. The surface growth will only accelerate, however, to a depth corresponding to the diffusion distance of the water.

To qualitatively model these data, a standard diffusion profile for one-dimensional diffusion of water into the particle was calculated for the time that the sample was placed in the most atmosphere $(80 \mathrm{~h}$ at room temperature). No account was taken of water re-distribution during the subsequent DSC/DTA scan. Lacking data for the diffusion coefficient of water in lithium disilicate glass, measured values for water diffusion in fused silica [26] were used to estimate a room temperature diffusion coefficient, giving $D \sim 2.8 \times 10^{-10} \mathrm{~cm}^{2} / \mathrm{s}$. The nucleation rate in hydrated glasses is known to be higher by almost an order of magnitude over that in non-hydrated ones [27]. Assuming that this is entirely due to a change in the mobility, a multiplicative constant for the growth velocity was taken to scale linearly with the water concentration, taking on values between one for the dry glass and ten for the fully hydrated glass (at the surface of the particle). Fig. $7(a)$ and (b) show the peak temperature and the peak height computed as a function of particle size. They are in fair agreement with the experimental data, showing a lower transi-

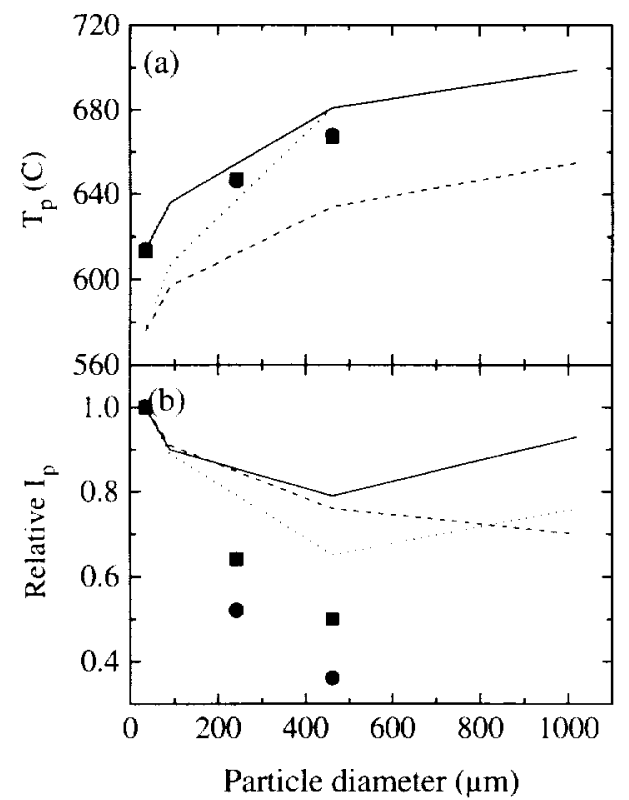

Fig. 7. The predicted effect of water on (a) the peak temperalure and (b) the relative peak height, $I_{\mathrm{r}}$. The points represent the experimental data; the lines represent the calculated results. Water treatment: dry samples (solid line, $\mathbf{a}$ ); surface hydrated sample where water diffused in from the surface (dotted line. $)$; fully hydrated sample where water was assumed uniform throughout the volume (dashed line). All calculations were performed for spherical glass particles quenched at $1{ }^{\circ} \mathrm{C} / \mathrm{s}$. The experimental error is of order the size of the data points.

tion temperature and a higher peak height in the hydrated glasses for the smaller particles. For the smaller particles, the calculations predict a difference in the peak temperatures and peak heights for the hydrated and dry glasses that is not observed experimentally. The reasons for this are not known; as for the Pt doped glasses, it may indicate a distribution of particle shapes different from those modeled here. Because the thickness of the hydrated layer expressed as a fraction of the particle diameter decreases with increasing particle size, the computed DSC/DTA parameters for the wet and dry glasses approach one another, and are in better agreement with the data. For comparison, the calculated DSC/DTA parameters for glasses that are fully hydrated throughout the volume are also shown in Fig. 7. Since both the surface and the volume growth velocities and the volume nucleation rate are increased by a factor of ten for all particle sizes, the 
parameters remain different for all particle sizes. While these calculations suggest that DSC/DTA data could be used to make rough estimates of the diffusion coefficient of water in glasses, a quantitative comparison would require an iterative calculation with a varying diffusion coefficient, modeling the change in both growth velocity and the nucleation rate as a function of distance from the surface.

\subsection{Non-isothermal nucleation treatments}

We have demonstrated previously that DSC /DTA scans following preannealing treatments can be used to estimate the temperature range for significant nucleation $[4,7]$. Here we compare model predictions for these studies with experimental data. Simulated

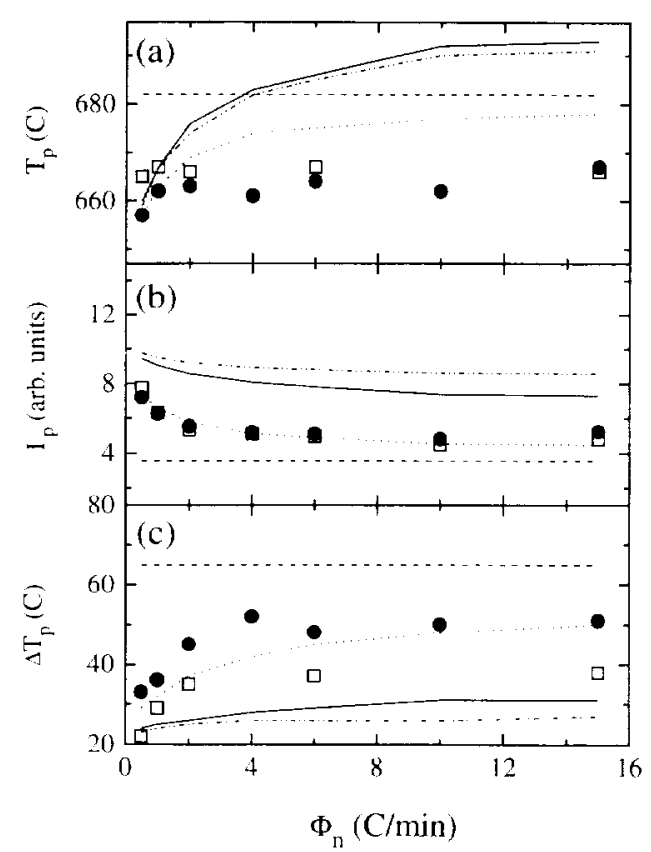

Fig. 8. The calculated effect on the DSC/DTA peak parameters of scanning through the nucleation zone at different rates: (a) the peak temperature (b) the peak height. $I_{F}$, and (c) the peak half-widh. Results are shown for infinite samples (dot-dol-dash line). finite-size correcled (FS) (solid line), surface crystallization (datshed line) and a combination of FS and surface corrections. (dotted line). Experimental data are also shown: (O) DSC data and ( $\square$ ) DTA data. All calculations were made for glass particles uniformly distributed between $425-500 \mu \mathrm{m}$ in diameter that had been quenched at $0.1^{\circ} \mathrm{C} / \mathrm{s}$. The experimental error is of order the size of the data points. as-quenched glasses were scanned first through the nucleation zone, from $400^{\circ} \mathrm{C}$ to $500^{\circ} \mathrm{C}$, at rates $\left(\Phi_{\mathrm{N}}\right)$ between 2 and $15^{\circ} \mathrm{C} / \mathrm{min}$ and were subsequently scanned through the crystallization peak. The crystallization scan rate, $\Phi_{\mathrm{C}}$, was typically $15^{\circ} \mathrm{C} / \mathrm{min}$.

Fig. 8 compares the predictions of the computer model for the key DSC /DTA peak parameters with experimental data as a function of the scan rate through the nucleation zone. Spherical particles of simulated glasses quenched at $0.1^{\circ} \mathrm{C} / \mathrm{s}$ with an average diameter of $462 \mu \mathrm{m}$ were assumed. The results from two independent DSC/DTA measurements made in different laboratories on these glasses are provided. The scatter between these data provides some indication of the expected experimental error. They indicate that the peak temperature and the peak height are the more robust parameters, better suited for quantitative comparison with model predictions. For comparison, calculated values for an infinite sample, for finite-size corrections only, for surface crystallization alone, and for combined surface and volume crystallization for finite-size particles are shown. In all cases, reasonable agreement with experimental observation is found. As expected. $T_{\mathrm{p}}$ and $\Delta T_{p}$ increase with increasing scan rate through the nucleation zone while $I_{\mathrm{p}}$ decreases. As the scan rate through the nucleation zone is decreased, the time for nucleation increases, resulting in a greater population of nuclei, causing an increase in the transformation rate. Although the agreement between the predicted and measured peak temperature is less good, it is still reasonable. It should be emphasized that no fitting parameters were adjusted for these calculations; the input data are those obtained from experimental measurements of the time-dependent nucleation rate and the macroscopic growth velocity as a function of temperature.

The calculated peak parameters for two particle sizes, $462 \mu \mathrm{m}$ and $1020 \mu \mathrm{m}$, of a glass quenched at $0.1^{\circ} \mathrm{C} / \mathrm{s}$ are compared with experimental data in Fig. 9. Spherical and ellipsoidal particles were considered. In all cases, the calculated results predict the correct trends, although they disagree quantitatively with the measurements. For the spherical particles (Fig. 9(a), (b) and (c)), the computed values for $T_{\mathrm{F}}$ show a larger change than is observed in the data, while the computed values for $I_{\mathrm{r}}$ and $\Delta T_{\mathrm{r}}$ are less than is observed. The agreement with experimental 


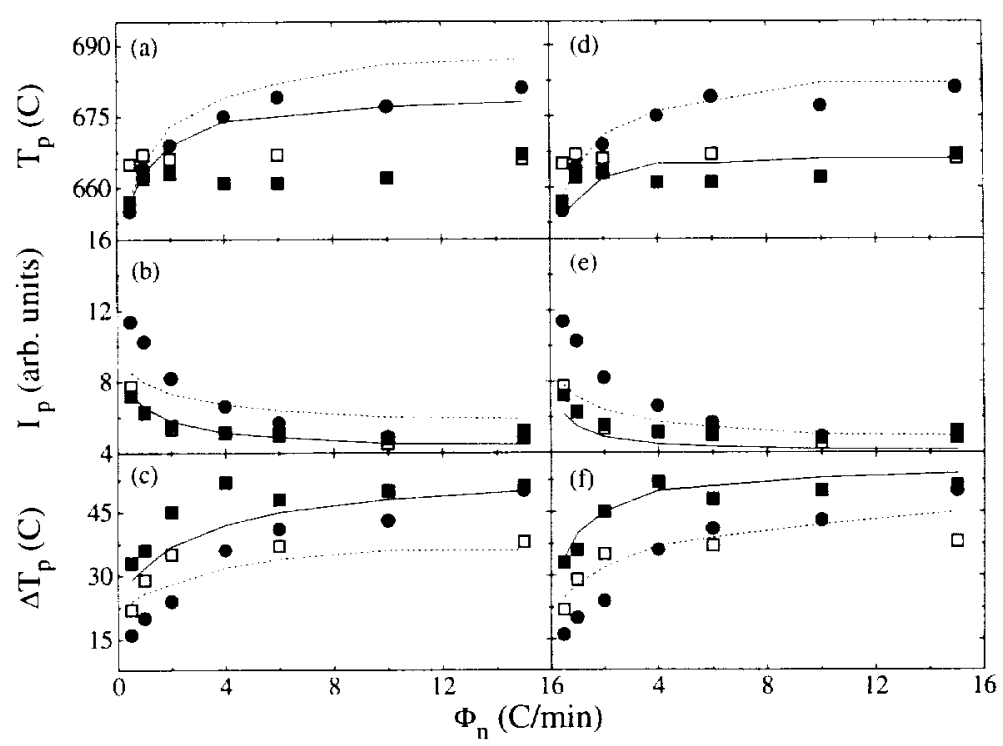

Fig. 9. The effect of scanning through the nucleation zone at different rates on the DSC/DTA peak parameters. Experimental data from DSC ( $)$ and from DTA ( $\square$ ) for particles with diameter $425-500 \mu \mathrm{m}$; DSC data from particles with diameter $850-1170 \mu \mathrm{m}(\mathrm{)})$. Calculations for spherical particles with diameter 425-500 $\mu \mathrm{m}$ (solid line) and 850-1170 $\mu \mathrm{m}$ (dashed line): (a) the peak temperature (b) the peak height, $I_{\mathrm{p}}$, and (c) the peak half-width. Corresponding results for ellipsoidal particles of eccentricity $\left(1, \frac{1}{2}, \frac{1}{2}\right)$ are shown in (d). (e). and (f) respectively. All glasses were quenched at $0.1^{\circ} \mathrm{C} / \mathrm{s}$. Calculations are shown for surface plus finite-size corrected volume crystallization. The experimental error is of order the size of the data points.

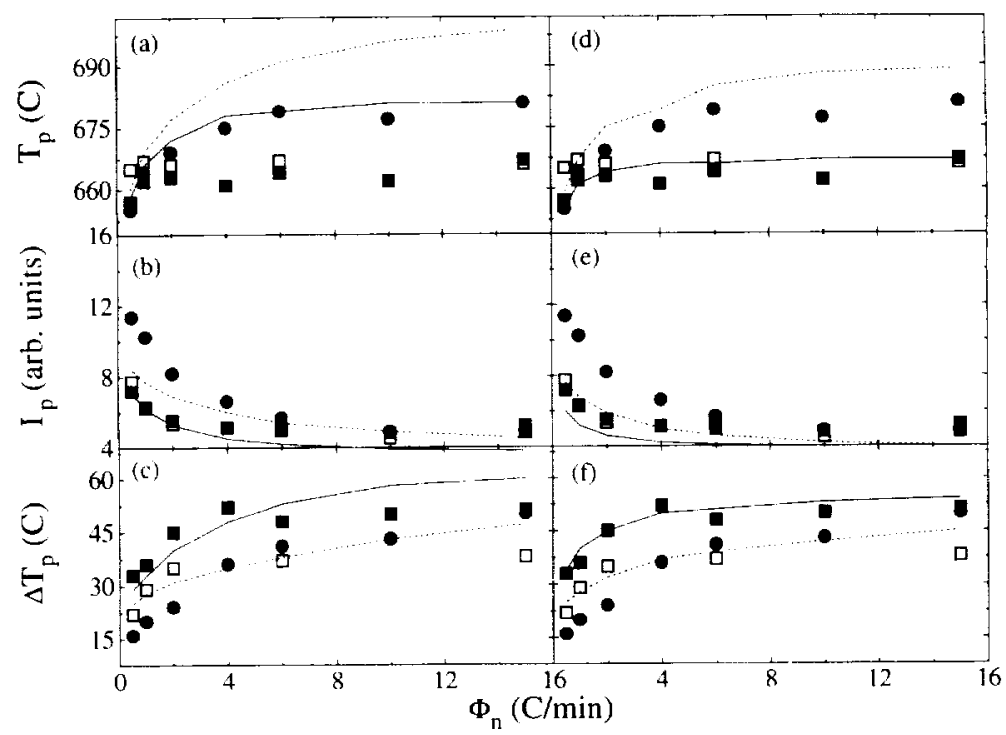

Fig. 10. The effect of scanning through the nucleation zone at different rates on the DSC/DTA peak parameters. Experimental data from DSC ( ) and from DTA ( $\square)$ for particles with diameter $425-500 \mu \mathrm{m}$; DSC data from particles with diameter $850-1170 \mu \mathrm{m}(\mathbf{)})$. Calculations for spherical particles with diameter $425-500 \mu \mathrm{m}$ (solid line) and $850-1170 \mu \mathrm{m}$ (dashed line): (a) the peak temperature (b) the peak height. $I_{\mathrm{n}}$, and (c) the peak half-width. Corresponding results for ellipsoidal particles of eccentricity $\left(1, \frac{1}{2}, \frac{1}{2}\right)$ are shown in (d), (e). and (f) respectively. All glasses were quenched at $1^{\circ} \mathrm{C} / \mathrm{s}$. Calculations are shown for surface plus finite-size corrected volume crystallization. 


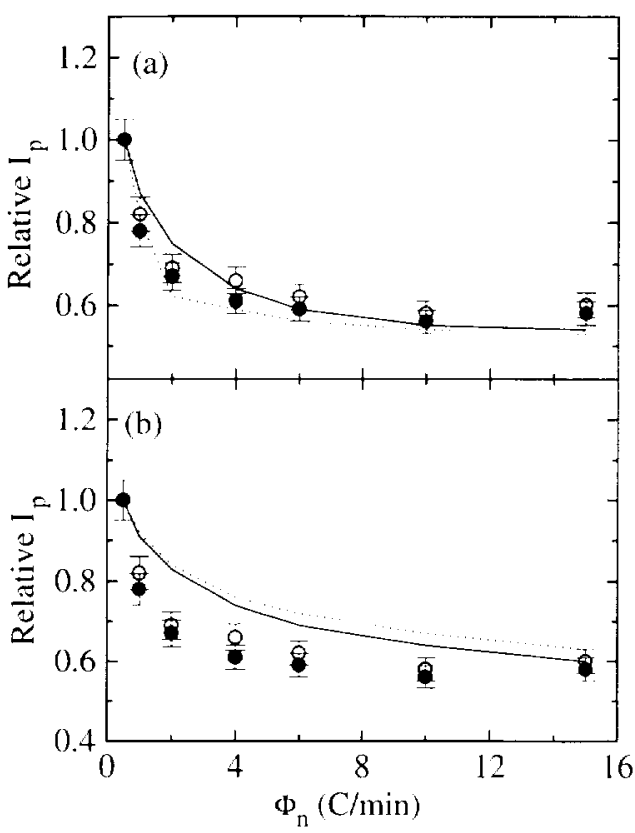

Fig. II. Calculated relative peak heights. $I_{\mathrm{p}}$. for glasses that hat been seanned through the nucleation zone (solid line) and for glasses held isothermally for a corresponding amount of time it the peak temperature of the steady-state nucleation rate (dashed line) compared with corresponding experimental data ( $(O)$ scanned glasses and $(O)$ isothermally treated glasses). Calculations in (a) were made atsuming time-dependent nucleation: steady-state nucleation was alssumed in (b). All glasses were quenched at $0.1^{\circ} \mathrm{C} / \mathrm{s}$. The experimental error is indicated by the error hars

data is significantly improved by assuming ellipsoidal particles instead of spherical ones (Fig. 9(d), (e) and (f)). although the predicted change in peak height as a function of particle size is smaller than is experimentally observed in both cases.

Since the experimental quench rates for the glasses were unknown, these calculations were repeated on simulated glasses that were quenched from the melt at rates of $1{ }^{\circ} \mathrm{C} / \mathrm{s}$ and $10^{\circ} \mathrm{C} / \mathrm{s}$. The results for the $10^{\circ} \mathrm{C} / \mathrm{s}$ quenched glasses were almost indistinguishable from those for the $1^{\circ} \mathrm{C} / \mathrm{s}$ glasses, which are shown in Fig. 10. The peak temperatures are fit slightly better by the glasses quenched at $0.1{ }^{\circ} \mathrm{C} / \mathrm{s}$, although the differences with calculations for glasses quenched at $1{ }^{\circ} \mathrm{C} / \mathrm{s}$ are small.

Fig. 11 shows the computed DSC/DTA peak heights for glasses that were either scanned at different rates through the nucleation zone $\left(400^{\circ} \mathrm{C}-500^{\circ} \mathrm{C}\right)$. or were annealed isothermally following the methods in Refs. [6.7]) at the nucleation peak temperature for a length of time equal to the total time required to scan through the range of significant nucleation $\left(425^{\circ} \mathrm{C}-500^{\circ} \mathrm{C}\right)$. As observed experimentally, the calculated peak heights for the two annealing treatments are identical, whether time-dependent (Fig. Il(a)) or steady-state nucleation (Fig. I1(b)) rates are assumed. in agreement with the experimental results. The reasons for this unexpected behavior are not obvious and are discussed further in the following sections. The experimentally observed changes in peak height with increasing scan rate and decreasing annealing time are sharper than predicted from the steady-state calculations. indicating a stronger dependence on the production of nuclei than is expected from the temperature dependence of the steady-state nucleation rate alone. Importantly. the experimental and calculated results are almost indistinguishable when time-dependent nucleation effects are included.

We have shown previously that the cluster distribution and hence the nucleation rate of as-quenched glasses is considerably decreased from the stealystate value $[6,14]$. Computer calculations of the subsequent nucleation behavior on non-isothermal heating predicted that the nucleation rate would rise above the steady-state value. peaking at a temperature higher than the peak of the steady-state rate [6]. Similar behavior is evident following the multi-step annealing treatments considered here. Fig. 12(a) shows the calculated nucleation rate at a cluster size. $n=310$ (well above the largest critical size, $n^{*}$, in the temperature range over which the transformation occurs) as a function of temperature for glasses scanned through the nucleation zone at $\Phi_{n}=4$ and $0.5^{\circ} \mathrm{C} / \mathrm{min}$ and for glasses annealed isothermally for corresponding times of 18.75 and $1.50 \mathrm{~min}$. In all cases. the nucleation rate rises above the steady-state rate at a temperature that is higher than that of the peak in the steady-state rate. Interestingly. two peaks in the nucleation rate are observed when $\Phi_{\mathrm{n}}=$ $0.5^{\circ} \mathrm{C} / \mathrm{min}$. The origin of this complex behavior is found by an examination of the temperature dependence of the cluster distribution. From Eq. (3) for the large clusters considered, where $n \gg n^{*} . N_{n, 1} \approx$ $N_{n+1, r}$, the nucleation rate is $I_{n, t} \approx N_{n, l}\left(k_{n}-k_{n}\right)$. which is in turn approximately $N_{t, l} k_{n}^{+}$since the forward rate is much greater than the backward rate 


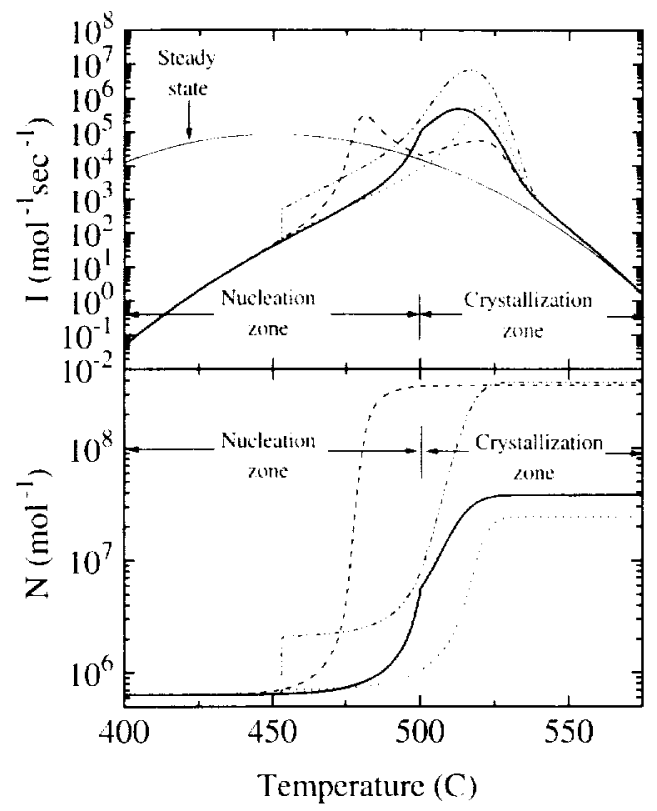

Fig. 12. (a) The calculated nucleation rate. $I$, as a function of temperature for glasses annealed in the nucleation zone: (b) the corresponding total number of nuclei. $N$. The solid and dashed lines are for samples scanned through the nucleation zone at $\Phi_{n}=4$ and $0.5^{\circ} \mathrm{C} / \mathrm{min}$. respectively: the dotted and dot-dot-dash lines are for samples annealed at the peak nucleation temperature for 18.75 and $150 \mathrm{~min}$ respectively. For comparison, the steadystate nucleation rate is also given. The nucleation sone (400)$50(0)^{\circ} \mathrm{Cl}$ and a portion of the crystallization $70 \mathrm{ne}(500)^{\circ} \mathrm{C}$ through the crystallization peak) are indicated.

there. The computer calculations, of course, follows Eq. (3) exactly; these simplifications are only made here to facilitate the discussion. During a DSC/DTA scan to higher temperature, therefore. the observed nucleation rate will result from a competition between the density. $N_{n,}$, which is evolving toward a temperature dependent steady state distribution, and the net ratte, which is becoming larger with increasing temperature because of the increasing atomic mobility. Considering first the case where $\Phi_{n}=$ $0.5^{\circ} \mathrm{C} / \mathrm{min}, N_{310, T(t)}$ reaches its maximum value near $480^{\circ} \mathrm{C}$ (Fig. 13). explaining the first peak in the nucleation rate. For this slow scan rate, the time spent at temperatures within the nucleation zone is sufficiently long that considerable relaxation toward the steady-state density occurs. Since $N_{310 . T(1)}$ decreases for all higher temperatures, the second. smaller, peak arises from the competition between the abrupt change in the rate of relaxation (and hence the rate of decrease of $N_{310 . T(1)}$ ) when the scan rate is changed from $\Phi_{\mathrm{n}}$ to $\Phi_{s}$, and the increased rate of change in $k_{n}^{4}$ resulting from the more rapid increase in temperature for $\Phi_{c}$. The effect becomes less dramatic as $\Phi_{n}$ approaches $\Phi_{\mathrm{c}}$, finally disappearing when $\Phi_{n}=\Phi_{\text {. }}$. For $\Phi_{\mathrm{n}}=4^{\circ} \mathrm{C} / \mathrm{min}$, only a kink is evident at the temperature for which the rate is increased to $\Phi_{\mathrm{c}}=15^{\circ} \mathrm{C} / \mathrm{min}$, reflecting the sudden change in rate constants. Now, the peak in the nucleation rate corresponds closely with that of the maximum in cluster density, pushed to higher temperatures because of the less complete relaxation occurring within the nucleation zone. This difference in degree of relaxation of the cluster density is also evident in calculated behavior for glasses annealed at the peak nucleation temperature $\left(450^{\circ} \mathrm{C}\right)$ for different times and subsequently scanned to higher temperatures at $15^{\circ} \mathrm{C} / \mathrm{min}$. The jump in the nucleation rate near $450^{\circ} \mathrm{C}$ for the glass annealed for $150 \mathrm{~min}$ is a result of the higher density. The more gradual change for the glass annealed for less time reflects the smaller degree of relaxation from the as-quenched distribution. At temperatures below $450^{\circ} \mathrm{C}$, the nucleation rate is independent of the annealing treatment. The observed temperature dependence reflects that of $k_{n}$; the cluster density remains virtually unchanged from the as-quenched value because of the low rate of relaxation.

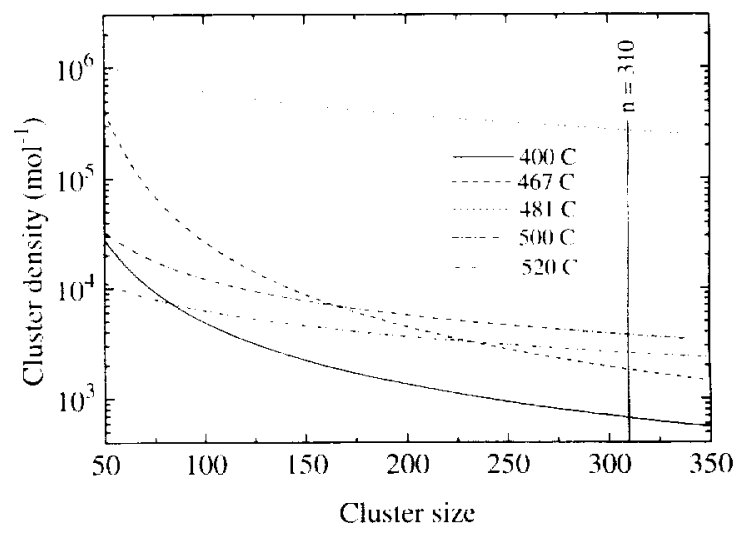

Fig. 13. $13 \mathrm{~A}$ portion of the cluster density for different temperalures for $\Phi_{11}=0.5^{\circ} \mathrm{C} / \mathrm{min}$ and $\Phi_{i}=15^{\circ} \mathrm{C} / \mathrm{min}$. The cluster size at which the nucleation rate is measured (Fig. 12(a)), $n=310$ ), is indicated. 
Fig. 12(b) shows the corresponding number of nuclei produced as a function of temperature. Glasses annealed more slowly (or for longer time) in the nucleation zone contain more nuclei, although the reason for this is far more complicated than could be ascertained from steady-state considerations. For example, for the non-isothermal case where $\Phi_{n}=$ $0.5^{\circ} \mathrm{C} / \mathrm{min}$, most of the nucleation occurs in the nucleation zone. Approximately the same number of nuclei are produced in the corresponding case of a $150 \mathrm{~min}$ isothermal anneal at the peak temperature of the steady-state nucleation rate, although they are nucleated at temperatures above the upper limit of the nucleation zone. Similar considerations apply for the higher values of $\Phi_{\mathrm{n}}$ and the corresponding isothermal treatments. Anneals in the nucleation zone, then, not only result in the production of nuclei, but also cause differing degrees of relaxation of the cluster distribution toward the steady-state distribution. Although there may be no significant production of nuclei at temperatures near the steady-state peak temperature, those clusters that are 'swept through' the distribution result in a larger nucleation rate at higher temperatures. It is only fortuitous that in this glass the isothermal anneals for three-quarters of the time required to scan through the nucleation zone produce a similar number of nuclei in the scanned samples.

These results further demonstrate the role of time-dependent nucleation phenomena in determining the stability and transformation behavior of glasses and other phases. They also show that simple interpretations of DSC/DTA non-isothermal transformation data are not possible. The success of the numerical model, however, indicates that it can be used to model these data to obtain quantitative $\mathbf{k i -}$ netic information.

\subsection{Analysis of peak profiles}

The differences in peak parameters discussed in the previous sections indicate that it should be possible to distinguish between various crystallization mechanisms based on a qualitative inspection of the peak shapes. For illustration, the computed DSC peak profiles for a heating rate of $15^{\circ} \mathrm{C} / \mathrm{min}$ of a sample consisting of $50 \mu \mathrm{m}$ diameter particles are shown in Fig. 14. These glasses were quenched from

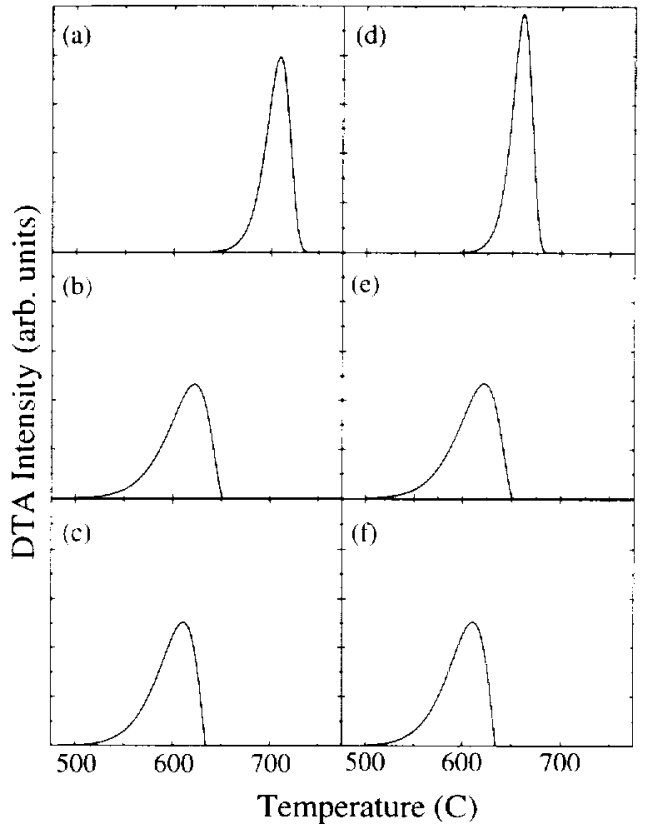

Fig. 14. The computed DSC/DTA peaks for $50 \mu \mathrm{m}$ diameter glass particles: (a), (d) infinite particle size; (b), (c) spherical particles; (c), (f) ellipsoidal particles with eccentricily $\left(1, \frac{1}{2}, \frac{1}{2}\right)$. Figures (a), (b) and (c) were for as-quenched glasses; (d), (e) and (f) were for glasses that were scanned at $1^{\circ} \mathrm{C} / \mathrm{min}$ between $400-500^{\circ} \mathrm{C}$ followed by a $15^{\circ} \mathrm{C} / \mathrm{min}$ scan from $500-900^{\circ} \mathrm{C}$. All calculations were performed for glasses quenched at $1{ }^{\circ} \mathrm{C} / \mathrm{s}$.

the melt at $1{ }^{\circ} \mathrm{C} / \mathrm{s}$ and had no preannealing treatments prior to the DSC/DTA experiment. The peak shape for the infinite sample (Fig. 14(a)) is qualitatively different from that obtained when finite particle-size effects and surface crystallization are included. The transformations for finite-sized spherical (Fig. 14(b)) and ellipsoidal (Fig. 14(c)) particles occur at lower temperatures and are spread out over a larger temperature range. Such differences could be interpreted by an appropriate model of the growth velocity, however. What might be interpreted experimentally is the degree of peak asymmetry. Peaks for the small particles show a much longer tail on the low temperature side of the peak. A comparison with the peaks from surface and volume crystallization alone shows that the peak shape for such small particles is dictated largely by surface crystallization. The predicted peak for ellipsoidal particles looks very similar to that for spherical particles, although the drop-off at high temperatures is sharper, reflect- 


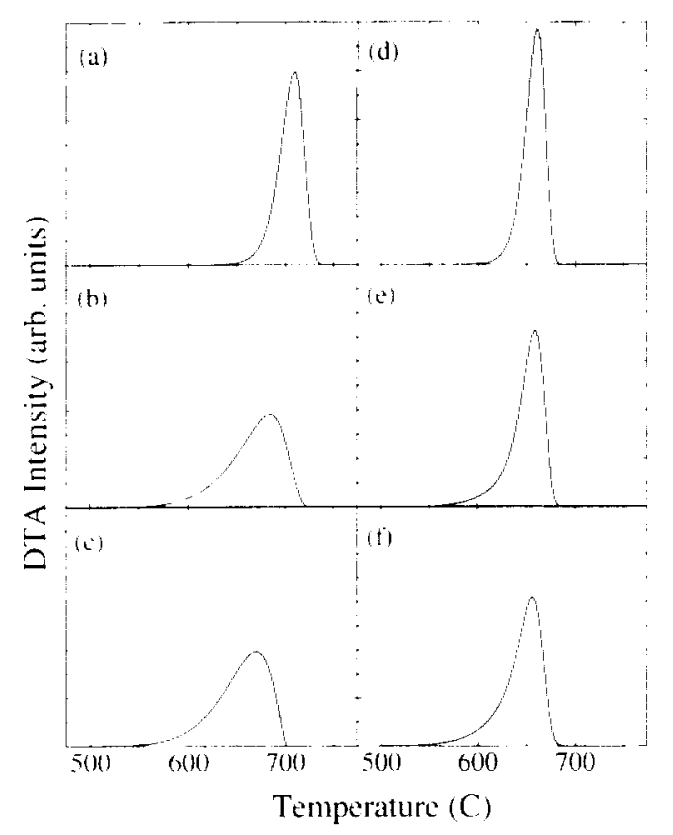

17ig. 15. The computed DSC D'TA peiks for 500 4 m diameler glats particles: (a). (d) infunte particle size: (b). (c) spherical

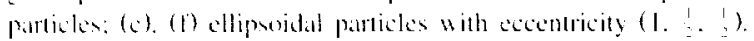
Figures (a).(1) and (o) were for ats-guenched glanses: (d). (e) and (1) were for glasses that were scanned at 1 "C / min hetween

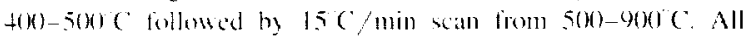
calcutations were pertermed for glasses quenched at $1 \mathrm{l}^{\prime} \mathrm{c} / \mathrm{s}$.

ing the greater impontance of surface crystallization due to the shorter distance between surfaces in two of the three dimensions. Fig. 14(d)-(f) show the predicted peiks for the infinite. and finite-sized spherical and ellipsoidal particles after the glass has been heated through the mucleation zone at $1{ }^{\circ} \mathrm{C} / \mathrm{min}$. That the peaks predicted for the smaller particles are virtually unchanged by the preannealing treatment reflects the dominance of surface crystallization. which is unaffected by annealing in the nucleation rone. since the surfice nucleation step is ignored here. The pealks for the infinitely large particles are shifted to lower temperatures because of the increased number of nuclei resulting from the preanne:al.

Fig. 15 shows the predicted peaks for larger. 500 $\mu \mathrm{m}$ diameter. particles of als-quenched glasses and of glasses that have been heated at $1{ }^{\circ} \mathrm{C} / \mathrm{min}$ through the nucleation zone. Taking both surface and volume eflects. the predicted DSC /DTA peaks for particles of the as-quenched glasses are still more asymmetric than for an infinite glass. showing a longer tail on the low temperature side of the peak. They are displaced to higher temperatures than for the smaller particles (Fig. 14(b) and (c)), reflecting the decreased importance of surface relative to volume nucleation. Annealing within the nucleation zone changes the DSC /DTA peak significantly for both the spherical and ellipsoidal particles. The peak temperatures are shifted down and the peak heights are increased, becoming more similar to the behavior of the infinite-sized particles. The increasing importance of volume nucleation and growth is also evidenced by the more symmetric peak shapes.

\section{Conclusions}

In conclusion we have presented a model for simulating polymorphic crystallization under nonisothermal and isothermal annealing conditions for particles of finite size. Surface and volume nucleation and growth. time-dependent nucleation rates and cluster-size dependent growth velocities are included. Predicted DSC/DTA scans of lithium disilicate glasses that had been heated at different rates although the temperature range where the steady-state nucleation rate is significant were compared with experimental results. The calculations were made using independently measured values for the nucleation and growth rates, the lemperature-dependent free energy difference between the glass and the crystal phases and the viscosity of the glass. Calculations were made as a function of particle size and particle shape and compared with the experimental dati. Glasses quenched at different rates were also investigated.

Calculated peak profiles were in very good agreement with measured data. given that no parameters were adjusted from their known values to improve the fits. The DSC/DTA peak height was shown to be sensitive to the contributions of the surface and volume and to the particle shape. A minimum in the peak height was predicted near particle radii of 200 $\mu \mathrm{m}$. due to the increasing importance of volume over surface crystitlization with increasing particle size. Fvidence for this minimum was found by examining the crystallization behavior of glasses as a 
function of Pt dopant level, a known heterogeneous nucleating agent that increases the contribution of internal growth. Future experiments on glass particles of carefully controlled size and shape will provide a more quantitative test of the model.

The good agreement strongly supports the model. It can be used to more accurately model DSC / DTA data than has been possible previously. Further, the numerical model provides a more complete insight into the fundamental processes of the devitrification. enabling better control of processing parameters and allowing estimates to be made of key kinetic parameters. The effects of time-dependent nucleation on phase stability and the transformation behavior and the ability of our numerical technique to model these quantitatively was demonstrated. The numerical model can also be used to design new experimental methods for analyzing calorimetric data. For example, these calculations demonstrate that if surface crystallization is known to be important in a system. the size below which it is the dominant mechanism can be determined from a plot of the height of the DSC/DTA peak versus particle size for particles of known shape. In those cases, the growth rate can be estimated as a function of temperature from one DSC/DTA experiment by assuming surface crystallization to fit the DSC/DTA peak.

\section{Acknowledgements}

The authors thank D.E. Day and M.C. Weinberg for useful discussions. This work was partially supported by NASA under contracts NAG 8-873. NAG 8-898 and NCC-849.

\section{References}

[1] C.S. Ray, D.E. Day, W. Huang. K.L. Narayan, T.S. Cull and K.F. Kelton, this issue p. 1.
[2] H.E. Kissinger. J. Res. Nat. Bur. Stand. 57 (1956) 217: Anal. Chem. 29 (1957) 1702: D.W. Henderson. J. Non-Cryst. Solids $30(1979) 301$

[3] H. Yinnon and D.R. Uhlmann, J. Non-Cryst. Solids 54 (1983) 253

[4] C.S. Ray and D.E. Day. J. Am. Ceram. Soc. 73 (1990) 430.

[5] X.J. Xu, C.S. Ray and D.E. Day, J. Am. Ceram. Soc. 74 (1991) 909.

[6] K.F. Kelton, J. Non-Cryst. Solids 163 (1993) 283

[7] K.F. Kelton, J. Am. Ceram. Soc. 75 (1992) 2449.

[8] K.F. Kelton and F. Spaepen, Acta Metall. 33 (1985) 455.

[9] M.C. Weinberg. J. Non-Cryst. Solids 1.34 (1991) 116.

[10] M.C. Weinberg and R. Kapral, J, Chem. Phys. 9l (1989) 7146.

[11] M.C. Weinberg. J. Non-Cryst. Solids 142 (1992) 126.

[12] K.F. Kelton, in: Solid State Physics, Vol. 45 (Academic Press. New York, 1991) pp. 75-177.

[13] K.F. Kelton, A.L. Greer and C.V. Thompson, J. Chem. Phys. $79(1983) 6261$

[14] K.F. Kelton and A.L. Greer, J. Non-Cryst. Solids 79 (1986) 295.

[15] K.F. Kelton and M.C. Weinberg, J. Non-Cryst. Solids 180 (1994) 17

[16] A.N. Kolmogorov, Iz. Akad. Nauk SSSR Ser. Fiz. 3 (1937) 3.5 .5$.

[17] W.A. Johnson and R.F. Meh]. Trans. AIME 135 (1939) 416

[18] M. Avrami. J. Chem. Phys. 7 (1939) 1103:8 (1940) 212; 9 (1941) 177 .

[19] L.E. Levine. K.L. Narayan and K.F. Kelton, submitted to J Mater. Res.

[20] JANAF Thermochemical Tahles. 2nd Ed. (US Department of Commerce, National Bureau of Slandards, Washington, DC. 1971).

[21] K.F. Kelton and A.I. Greer, Phys. Rev. B38 (1988) 10089; A.L. Greer and K.F. Kelton, J. Am. Ceram. Soc. 74 (1991) 1015

[22] K. Matusita and M. Tashiro, Jpn. J. Ceram. Assoc. 81 (1973) 500 .

[2.3] K. Matusitat and M. Tashiro, J. Non-Cryst. Solids 11 (1973) 471.

[24] M.F. Barker, T.H. Wang and P.F. James, Phys. Chem Glasses 29 (1988) 240); J. Deubener, R. Brückner and $\mathrm{M}$ Starnitzke. J. Non-Cryst. Solids 163 (1993) 1.

[25] K.L. Narayan. K.F. Kelton and C.S. Ray, J. Non-Cryst. Solids $195(1996) 148$.

[26] R.H. Doremus, in: Modern Aspects of the Vitreous State Vol. II (Butterworth, London. 1962) pp. 1-71.

[27] C.J.R. Gonzalez-Oliver, P.S. Johnson and P.F. James, J Mater. Soi, 14 (1979) 1159. 



\section{Journal of Non-Crystalline Solids}

\section{Instructions to Authors}

Submission of papers

Manuscripts (one original + two copies), should be sent to the editors or any one of the regional editors. The address of the editors is as follows:

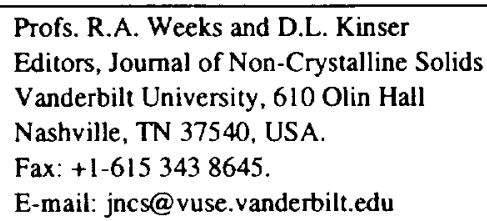

Original material: On submission, authors are asked to confirm that the manuscript is not being simultaneously considered for publication elsewhere and that all authors have approved the manuscript and take full responsibility for its contents and so state in their letter of transmittal.

\section{Types of contributions}

Original research papers, reviews, letters to the editor and commentaries are welcome. They should contain an Abstract (of up to 200 words) and a Conclusions section which, particularly in the case of theoretical papers, translates the results into terms readily accessible to most readers.

Letters should be no longer than six double-spaced typed pages. They will be given priority in both the refereeing and production processes. The faster production schedule will preclude sending proofs of letters to authors.

\section{Manuscript preparation}

All manuscripts should be written in good English. The paper copies of the text should be prepared with double line spacing and wide margins, on numbered sheets. See notes opposite on electronic version of manuscripts.

Structure. Please adhere to the following order of presentation: Article title, Author(s), Affiliation(s), Abstract, PACS codes, Main text (Introduction; Experimental procedures or constraints on theory; Results; Discussion; Conclusion. Sections and sub-sections must be clearly numbered according to the Joumal style), Acknowledgements, Appendices, References, Figure captions, Tables. Corresponding author. The name, complete postal address, telephone and fax numbers and the e-mail address of the corresponding author should be given on the first page of the manuscript.

References. References to other work should be consecutively numbered in the text using square brackets and listed by number in the Reference list. Please refer to past issues of the Journal for examples.

Symbols/Units: Authors should follow the Symbols and Units Nomenclature Commission of the I.U.P.A.P. (Physica 146A (1987) $1-68)$.

\section{Illustrations (figures)}

Illustrations should also be submitted in triplicate: one master set and two sets of copies. The line drawings in the master set should be original laser printer or plotter output or drawn in black india ink, with careful lettering; lettering should $2 \mathrm{~mm}$ in height after reduction for printing. The photographs should be originals, with somewhat more contrast than is required in the printed version. The top edge should be indicated on the back. They should be unmounted unless part of a composite figure. Any scale markers should be inserted on the photograph itself, not drawn below it.
Colour plates. Figures may be published in colour, if this is judged essential by the editor. The publisher and the author will each bear part of the extra costs involved. Further information is available from the publisher

\section{Classification codes/keywords}

Please supply one to four classification codes (PACS and/or MSC) and up to six keywords of your own choice that describe the content of your article in more detail.

\section{After acceptance}

Notification. You will be notified by the Editor of the journal of the acceptance of your article and invited to supply an electronic version of the accepted text, if this is not already available.

Copyright transfer. In the course of the production process you will be asked to transfer the copyright of the article to the publisher. This transfer will ensure the widest possible dissemination of information.

IMPORTANT: When page proofs of the accepted manuscript are made and sent to authors, this is in order to check that no undetected errors have arisen in the typesetting (or file conversion) process. Only printers' errors may be corrected; no changes in, or additions to, the edited manuscript will be accepted. In the case of extended changes, the authors will be required to pay part of the extra costs involved.

\section{Electronic manuscripts}

The publisher welcomes the receipt of an electronic version of your accepted manuscript. If there is not already a copy of this (on diskette) with the joumal editor at the time the manuscript is being refereed, you will be asked to send a file with the text of the accepted manuscript directly to the Publisher by e-mail or on diskette (allowed formats $3.5^{-}$or $5.25^{\circ}$ MS-DOS, or $3.5^{\circ}$ Macintosh) to the address given below. Please note that no deviations from the version accepted by the Editor of the joumal are permissible without the prior and explicit approval by the Editor. Such changes should be clearly indicated on an accompanying printout of the file.

\section{Author benefits}

No page charges. Publishing in Journal of Non-Crystalline Solids is free.

Free offprints. The corresponding author will receive 50 offprints free of charge. An offprint order form will be supplied by the publisher for ordering any additional paid offprints.

Discouns. Contributors to Elsevier Science journals are entitled to a $30 \%$ discount on all Elsevier Science books.

Further information (after acceptance)

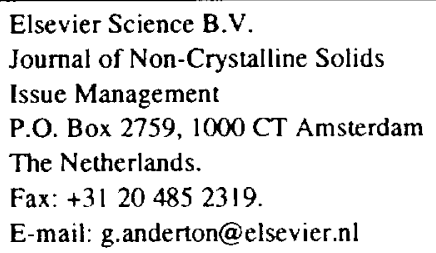

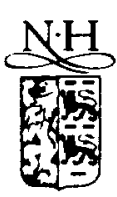

North-Holland, an imprint of Elsevier Science 
